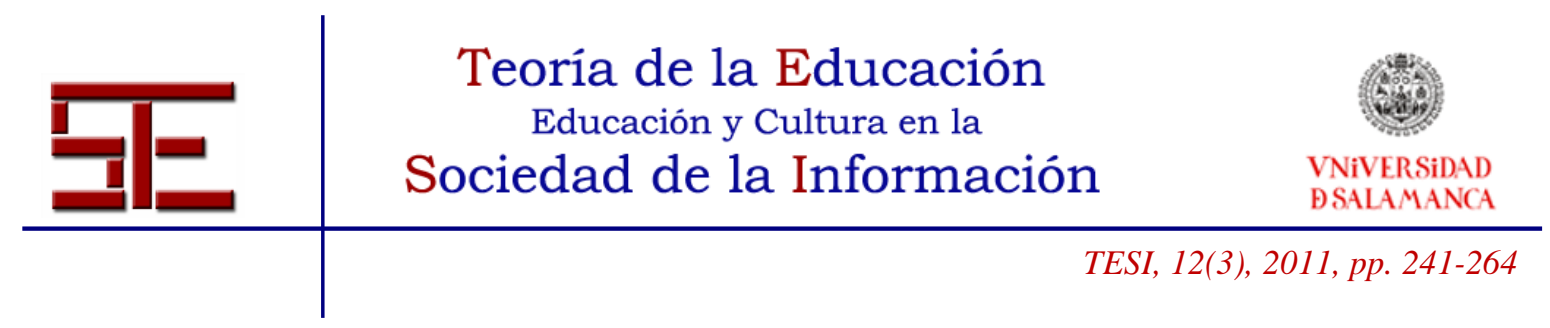

\title{
INICIATIVAS EDUCATIVAS PARA UNA SOCIEDAD DE LA INFORMACIÓN SOSTENIBLE
}

Resumen: El número de matriculaciones en grados relacionados con las Tecnologías de la Información, especialmente Informática y Telecomunicaciones, han sufrido un continuo descenso en la última década, aún más profundo si cabe en el colectivo femenino. Esta situación preocupa a empresas, gobiernos y universidades, ya que si la tendencia persiste no habrá suficientes profesionales de las Tecnologías de la Información y las Comunicaciones (TIC) que puedan mantener el ritmo de innovación y mejora competitiva en los países occidentales en un futuro cercano.

En este artículo se pretenden analizar las razones que han llevado a esta situación desde el punto de vista de los jóvenes y los centros de educación superior, con especial atención en los factores que afectan a las mujeres. Y se da una perspectiva de las distintas iniciativas innovadoras que se están llevando a cabo por parte de las Universidades y Administraciones públicas para acercar la realidad de la profesión informática a toda la sociedad y contrarrestar esta tendencia.

Palabras clave: Matriculación; mujeres en Ingeniería; Ingeniería Informática; innovación educativa.

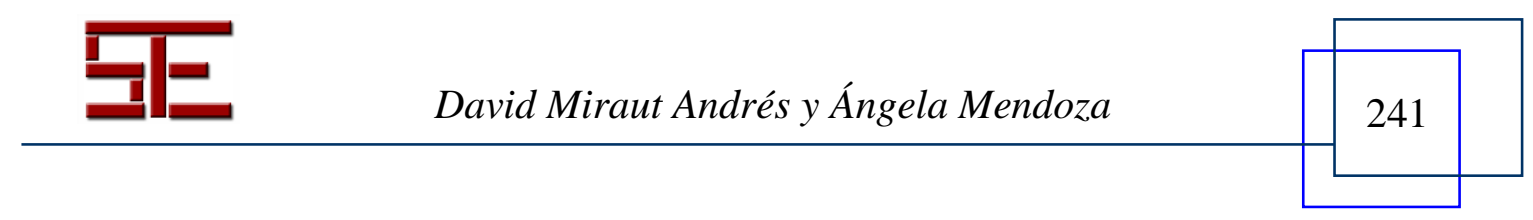




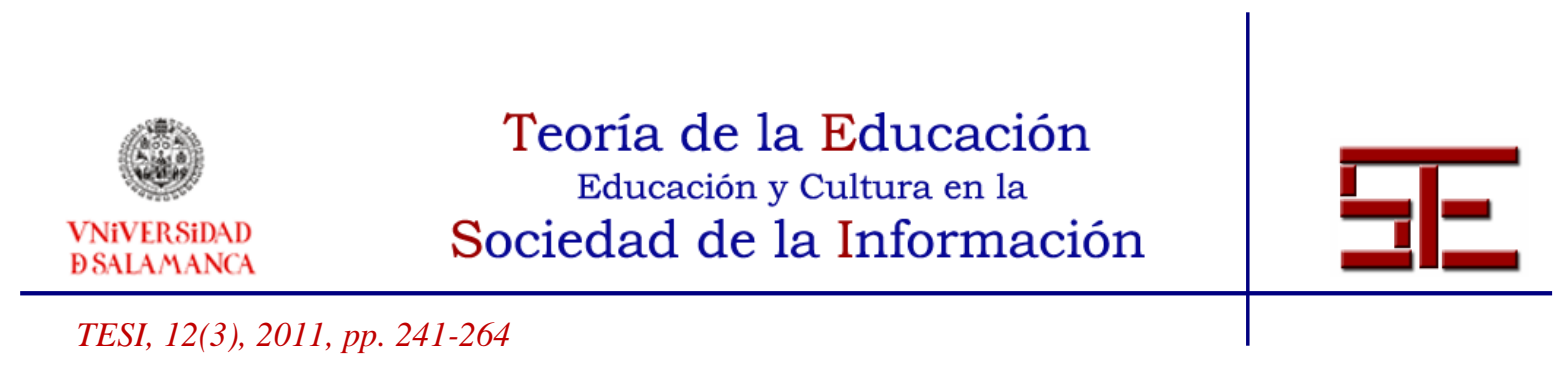

\title{
EDUCATIONAL INITIATIVES FOR A SUSTAINABLE INFORMATION SOCIETY
}

\begin{abstract}
Enrollment in degrees related to Information Technology (Computer Science and Telecommunications) has suffered a steady decline in the last decade, especially in the case of women. Industry, Government and Academia are concern. In a near future, we can reach to a point where there will be not enough professionals to cover Information Technology and Communications (ICT) positions, if this situation does not change. So, the pace of innovation and competitiveness could not be maintained in Western countries.

This paper analyzes some of the reasons why this increasing demand for ICT engineers is not tuned to the present trend of career choices among young people. And it provides an overview of the various innovative initiatives that are being carried out by all agents to show how ICT profession actually is to society in order to counteract this trend.
\end{abstract}

Keywords: Enrollment; women in Engineering; Computer Science Degrees; educational innovation.

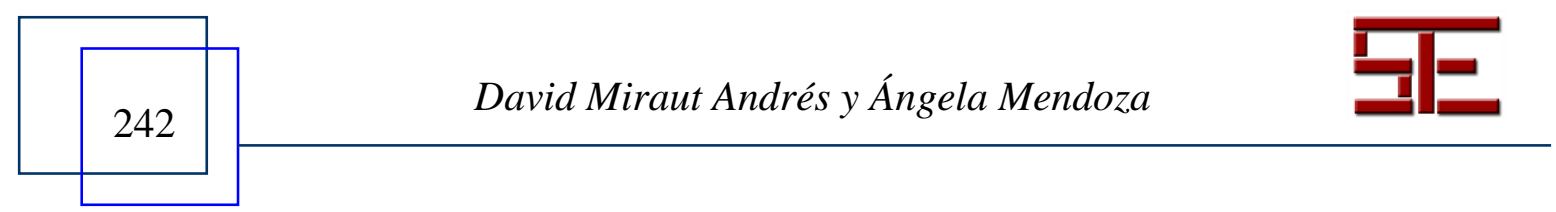




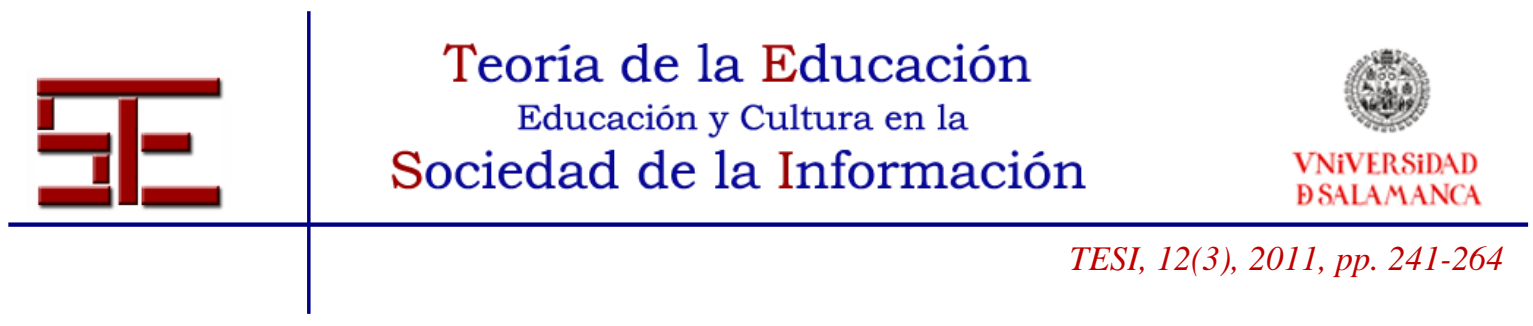

\title{
INICIATIVAS EDUCATIVAS PARA UNA SOCIEDAD DE LA INFORMACIÓN SOSTENIBLE
}

Fecha de recepción: 20/04/2011; fecha de aceptación: 26/10/2011; fecha de publicación: 30/11/2011

\author{
David Miraut Andrés \\ david.miraut@urjc.es \\ Universidad Rey Juan Carlos \\ Ángela Mendoza \\ angela.mendoza@urjc.es \\ Universidad Rey Juan Carlos
}

\section{1.- INTRODUCCION}

La información y su intercambio, pilares básicos de nuestra sociedad, han estado presentes desde el comienzo del pensamiento simbólico y el habla en las comunidades primitivas. La modernización hacia la actual sociedad de la información y su despliegue ha sido posible gracias a las tecnologías digitales que han favorecido el tratamiento, procesado y distribución de esta materia prima en las actividades culturales y económicas, de una forma rápida, automática y a un coste bajo gracias a la economía de escala.

Los ordenadores y las redes de comunicación se han convertido en herramientas que nos permiten aumentar nuestras habilidades. De un modo semejante a como las hachas y los arados nos permitieron ser más productivos en el pasado, ahora los dispositivos electrónicos mejoran nuestra percepción y amplían la capacidad de nuestras mentes. Tal y como Steve Jobs ilustró magistralmente con un curioso símil en una entrevista hace ya 30 años (Jobs, 1980):

¿Qué es un ordenador personal?

Permítanme contestar con la analogía entre la bicicleta y el cóndor.

Hace unos cuantos años, leí un estudio -creo que en el Scientific American- sobre la eficiencia de la locomoción de diversas especies de la tierra, incluyendo el hombre. El estudio determinaba qué especies eran las más eficientes cuando se trataba de recorrer la distancia desde $\mathrm{A}$ hasta $\mathrm{B}$ consumiendo la mínima energía. Ganó el cóndor. El Hombre resultó no ser demasiado impresionante, quedando más o menos en el último tercio de la lista. Pero alguien tuvo la ocurrencia de hacer la prueba al hombre montado en bicicleta. Entonces el hombre resultó ser dos veces más eficiente que el cóndor.

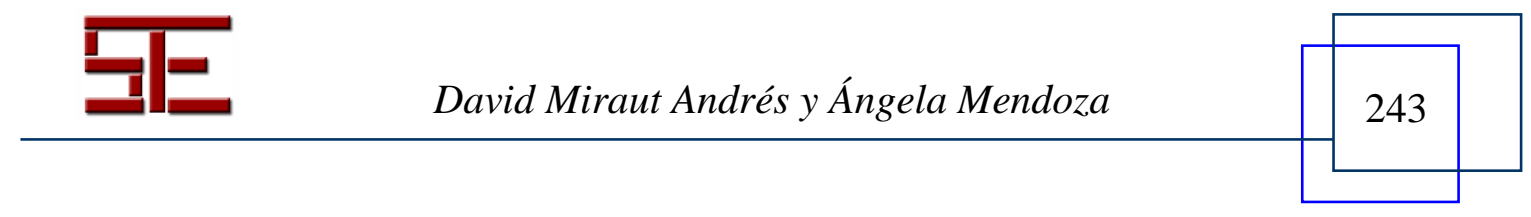




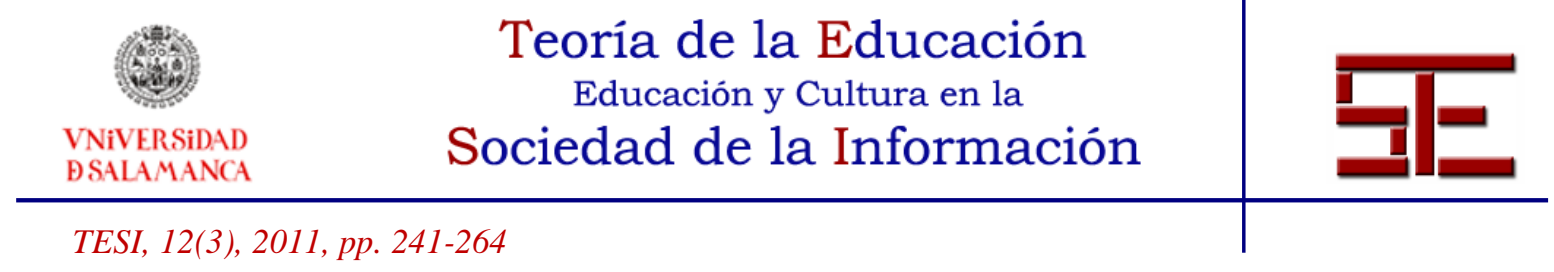

\begin{abstract}
Esto ilustra la habilidad del hombre como creador de herramientas. Cuando el hombre inventó la bicicleta, creó una herramienta que amplificaba cierta parte de su inherente capacidad. Por eso me gusta comparar al ordenador personal con la bicicleta.

El ordenador de Apple es -si quieren llamarlo así- la bicicleta del siglo XXI, al ser una herramienta que amplifica cierta parte de nuestra inteligencia. Llega a desarrollarse una relación especial entre la persona y el ordenador, que finalmente mejora la productividad a nivel personal.
\end{abstract}

Precisamente ahora, que la informática ha demostrado ser un factor clave en la mejora de la productividad tanto en nuestros procesos industriales y de servicios, como en el bienestar de la sociedad; en un momento en el que la espiral de innovación y adopción de la tecnología se mueve cada vez más rápido acompañada de los beneficios de la economía de escala; y cuando la demanda de profesionales en las Tecnologías de Información y las Comunicaciones (TIC) no deja de crecer dado que el sector no se ve influenciado por la crisis; e incluso algunos científicos sueñan con la singularidad tecnológica (Vernor, 1993), lo cierto es que nos encontramos ante una peligrosa paradoja que ha generado una gran cantidad de artículos:

El número de matriculaciones en grados relacionados con las Tecnologías de la Información, especialmente Informática y Telecomunicaciones, ha sufrido un continuo y profundo descenso en la última década.

Un informe realizado por la Computer Research Association (CRA), basado en una serie de encuestas llevadas a cabo por el Educational Research Institute of California, dio la voz de alarma al demostrar que el número de estudiantes de titulaciones relacionadas con la informática había descendido un $60 \%$ en el periodo entre los años 2000 y 2004 en todo el territorio estadounidense (Patterson, 2005). Si nos fijamos en el porcentaje de alumnas, los números son todavía más preocupantes, ya que su proporción se redujo en casi un 80\% entre 1998 y 2004.

Tras la crisis de la burbuja de las "punto com" entre los años 2000 y 2002, cada vez menos estudiantes se han interesado por las ingenierías relacionadas con Informática y Sistemas de la Información (Denning \& McGettrick, 2005; Lomerson \& Pollacia, 2006). Y esta tendencia no ha mejorado en los últimos cuatro años, en los que la matriculación ha seguido reduciéndose en un $40 \%$ en EE. UU. (Becerra-Fernández et al., 2010), y es muy similar en el resto de los países occidentales.

El Instituto de Estadística de la UNESCO indica en sus informes que muchos países europeos (como España, Francia, Alemania o Italia) sufren también un descenso pronunciado en las matriculaciones de carreras relacionadas con las ingenierías,

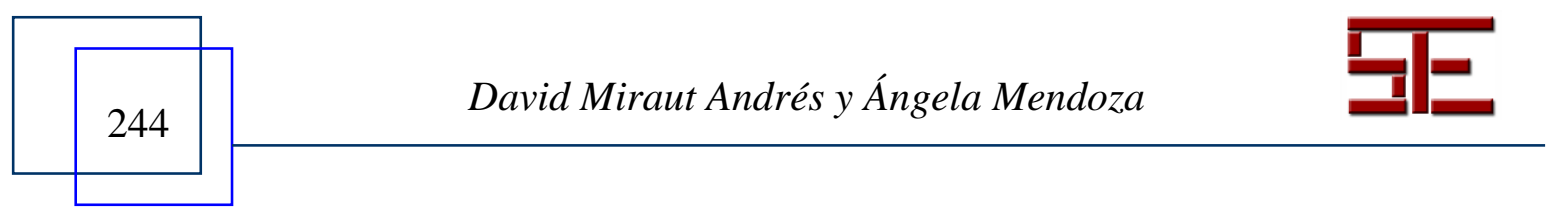




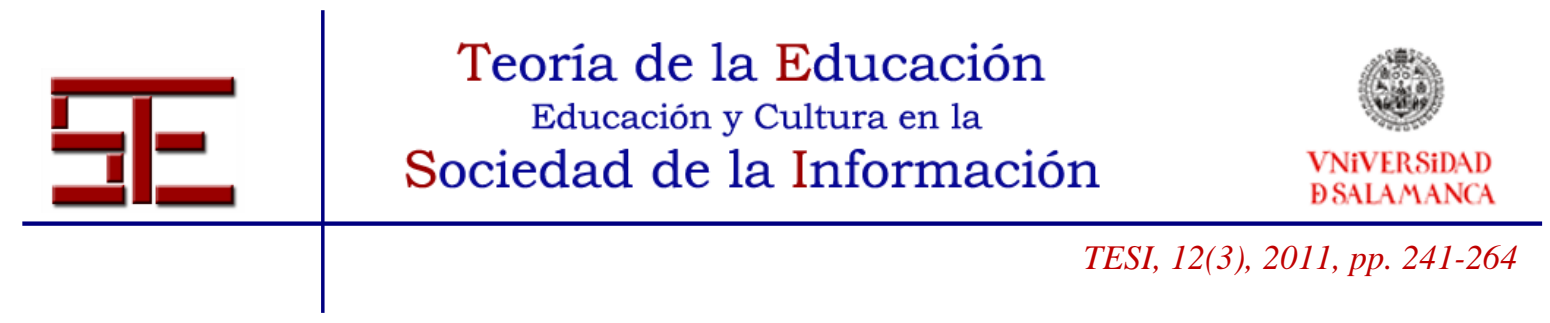

especialmente las TIC, donde el número de estudiantes se ha reducido a la mitad en la última década, mostrando una tendencia muy parecida a la estadounidense (Vesgo, 2008).

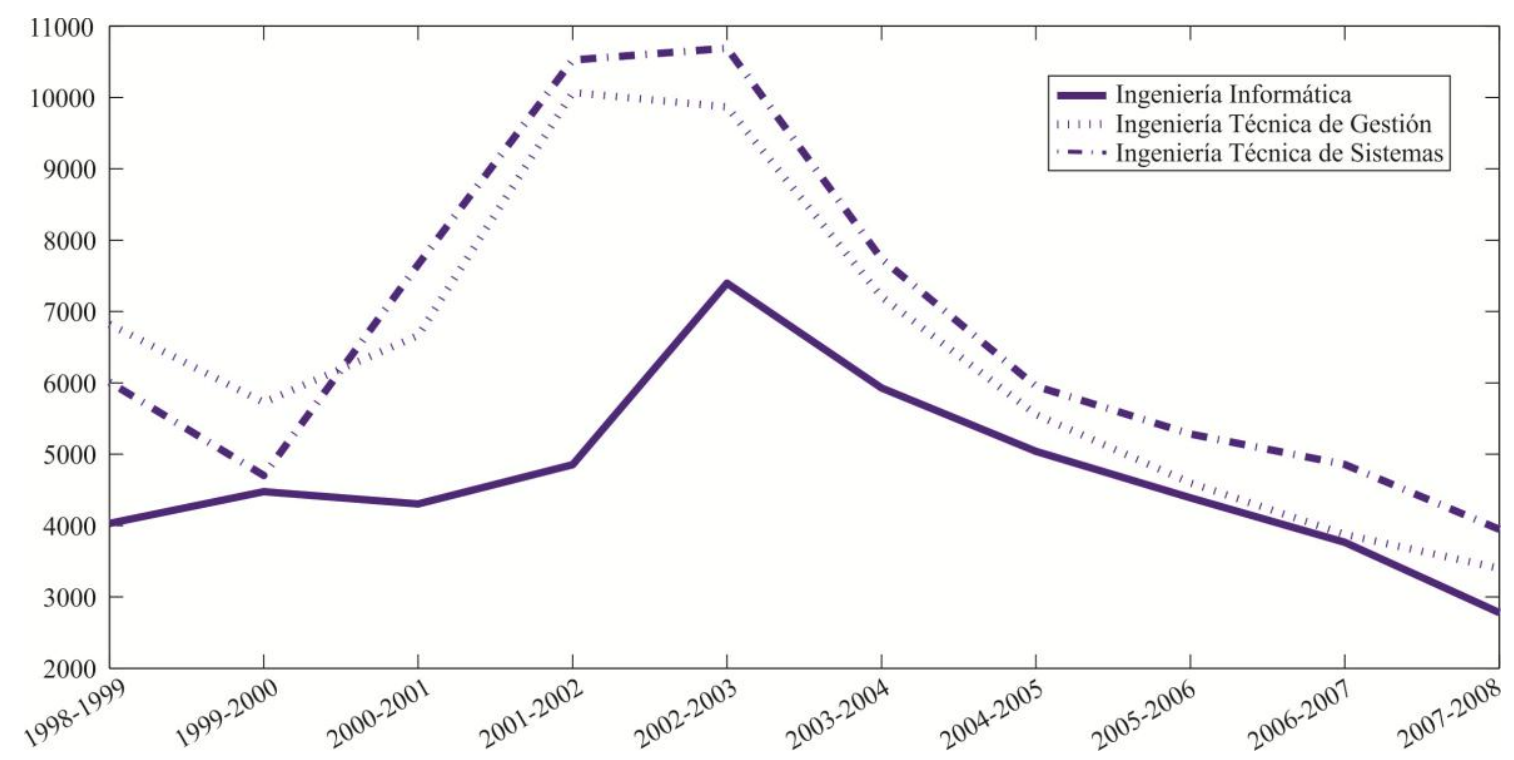

Figura 1. Nuevos alumnos en ingenierías informáticas por curso académico en las universidades públicas españolas. Extraído de la base de datos del INE (2010).

Si nos centramos en el caso de España, las cifras de matriculaciones son desalentadoras en el conjunto de las universidades públicas, como reflejan los datos del Instituto de Estadística que se ilustran en las Figuras 1 y 2 para las ingenierías informáticas (no se ha incluido el curso 2008-2009 al aparecer una gran diversidad de grados y titulaciones dobles relacionados con la informática al aplicarse el Espacio de Educación Superior, cuyos datos no está correctamente reflejados en la base de datos pública). Siendo muy similares a las que se obtienen con los datos de las ingenierías de Telecomunicaciones. En las gráficas se observa un pico en el número de matriculaciones que -curiosamentecoincide con el final de la crisis de las "punto com" (en la que desaparecieron casi 5000 empresas tecnológicas que operaban a través de Internet, bien por haberse fusionado o bien simplemente por quiebra), y desde entonces el número de nuevos alumnos ha descendido de forma continua. Cabría pensar que el descenso de las matriculaciones podría estar correlacionado con el demográfico, por el que ha disminuido el número de alumnos en educación secundaria, sin embargo, podemos comprobar en la Figura 3 que

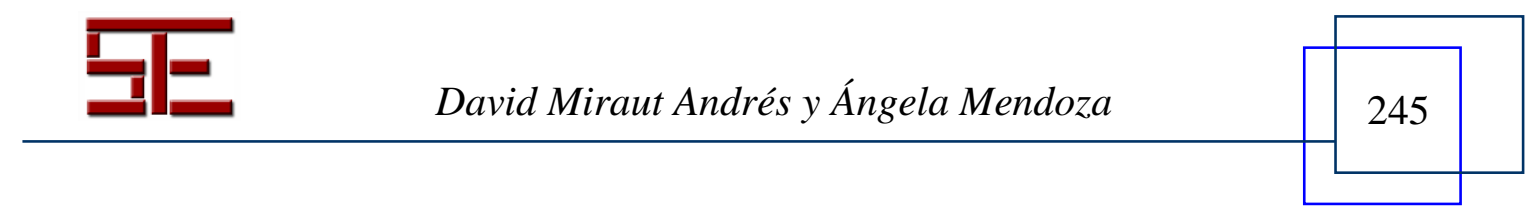




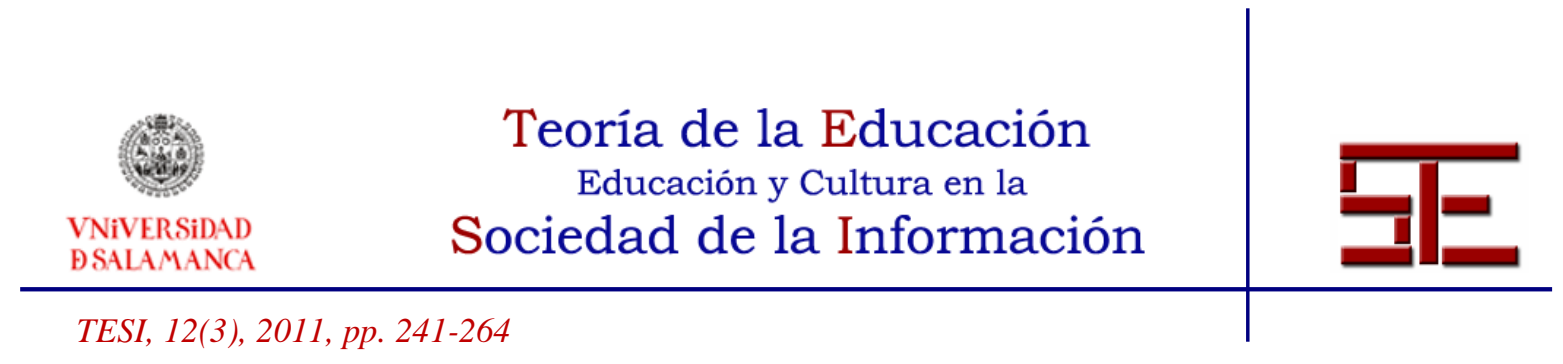

el número de alumnos que aprueban selectividad (en ambas convocatorias) cada año se ha reducido de una forma que poco tiene que ver con la caída de la matriculación en esta disciplina.

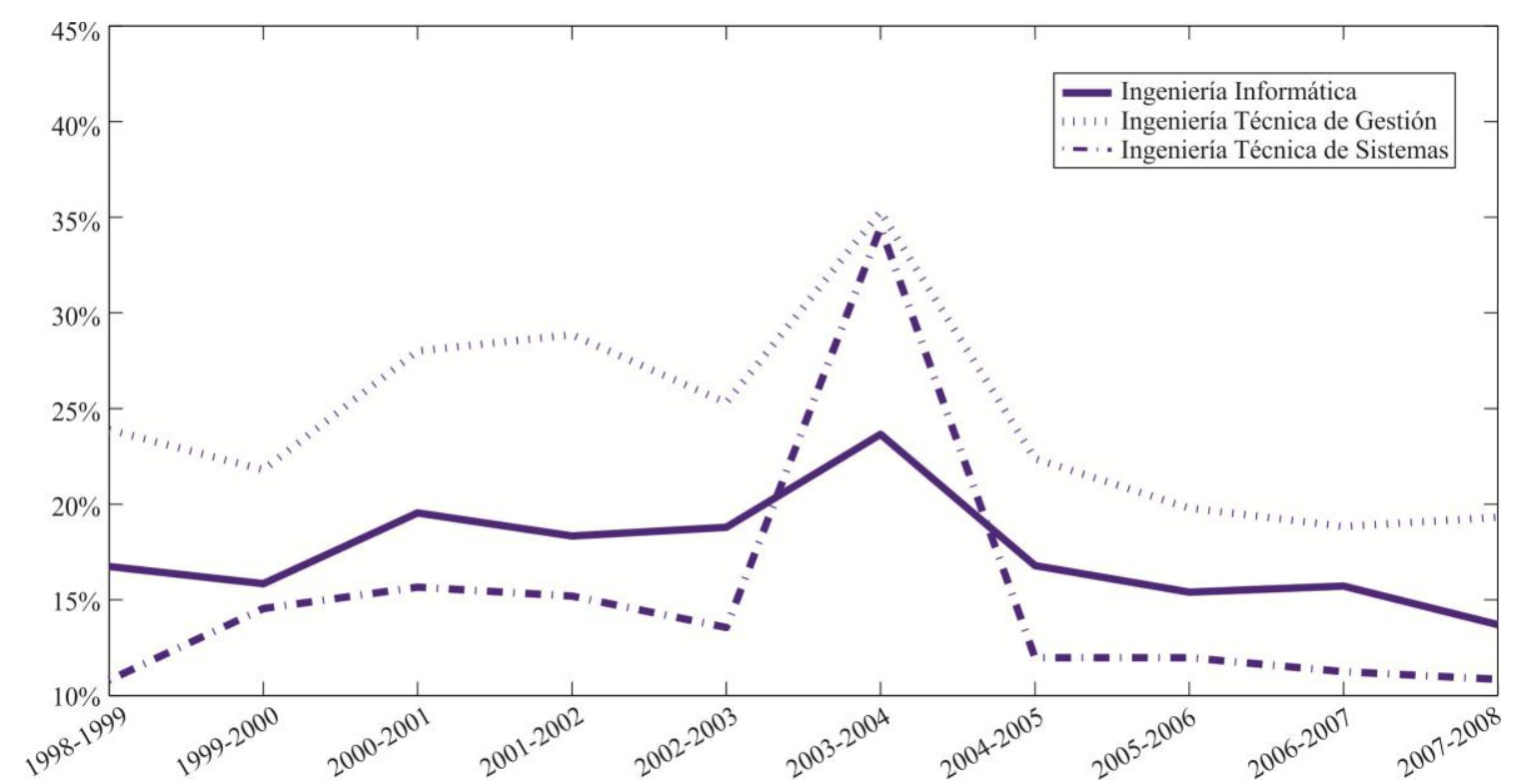

Figura 2. Porcentaje de matrículas de nuevas alumnas en ingenierías informáticas por curso académico en las universidades públicas españolas. Extraído de la base de datos del INE (2010).

Igualmente preocupante es el descenso en el porcentaje de mujeres en estas ingenierías, que parecen tener asociado un estereotipo fuertemente masculino. Un descenso que no tiene relación con los resultados en selectividad, en el que el alumnado femenino mantiene un porcentaje estable de los aprobados en torno al 58\%. En la Figura 2 se muestra la tendencia en las universidades públicas españolas, que si bien no es tan acusada como la estadounidense, tiene características parecidas.

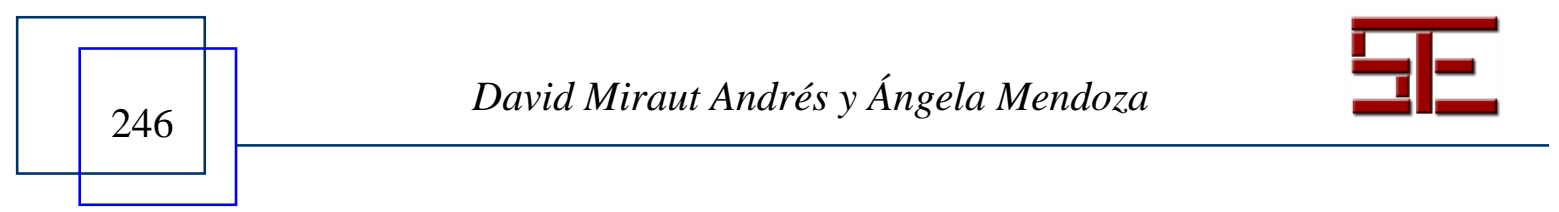



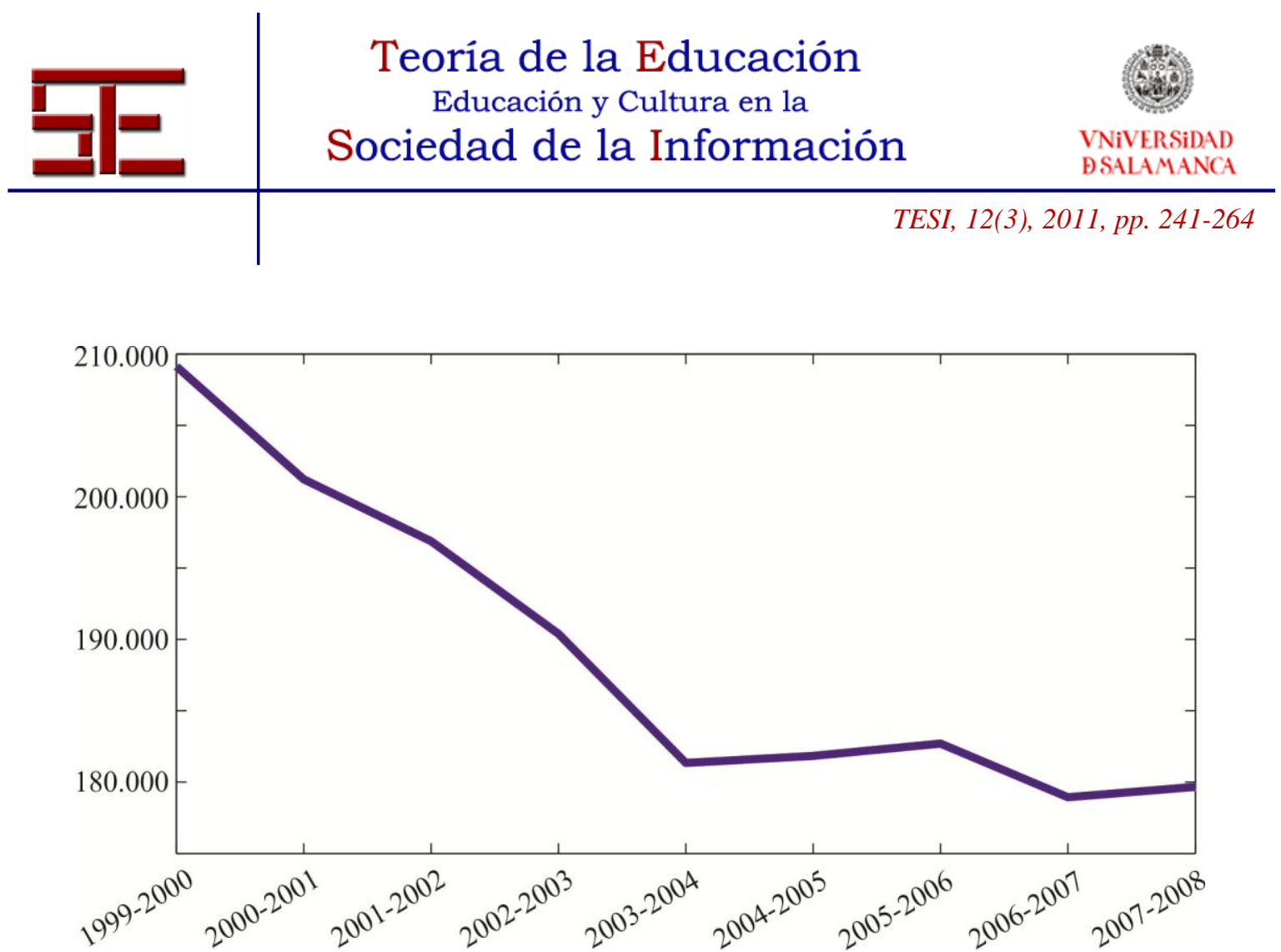

Figura. 3 Número de alumnos que han aprobado selectividad por curso académico.

Extraído de la base de datos del INE (2010).

El Bureau of Labor Statistics en EE.UU. es claramente optimista, prevé un incremento en la demanda laboral en el sector de la informática por encima del $40 \%$ en la próxima década (frente al 10\% medio previsto para el resto de la industria) con una retribución y prestigio notablemente altos para los profesionales especializados. La demanda estimada será especialmente fuerte en los puestos que requieren conocimientos estratégicos, como son los relacionados con Ingeniería de Computadores y análisis y diseño de sistemas de información (Denning \& McGettrink, 2005; Bureau, 2010).

La creciente demanda de ingenieros TIC no se corresponde con la tendencia actual en la elección de las carreras entre los jóvenes de los países occidentales. Esta situación preocupa a gobiernos, industria y universidades por igual en los países occidentales, ya que no será posible mantener el ritmo de innovación en nuestra sociedad a medio plazo si no hay profesionales que la puedan llevar a cabo. Es más, la externalización de servicios está llevando a países con economías emergentes una parte importante de la producción TIC, con lo que otros países están adquiriendo el know-how para competir en los campos que aportan los mayores beneficios en productividad y valor añadido.

Así, el equipo de gobierno de la administración Obama en EE.UU., el de Merkel en Alemanía y el de Sarkosi en Francia han tomado cartas en el asunto en sus respectivos

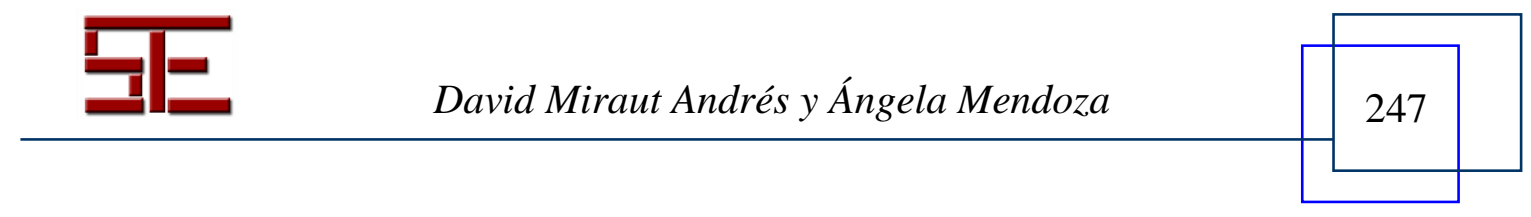




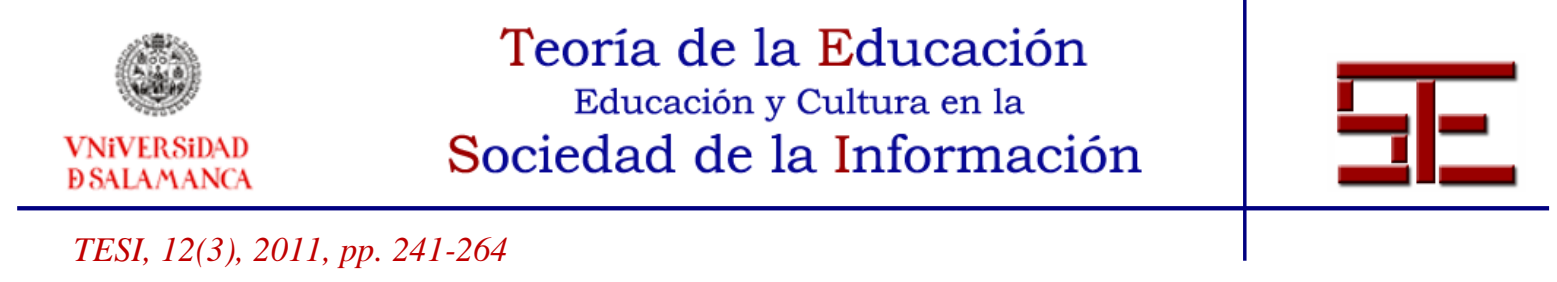

países y han iniciado un conjunto de actividades y certámenes sin precedentes para despertar la curiosidad de los jóvenes y que de forma atractiva tomen un papel activo en el desarrollo de la tecnología. Grandes empresas, como Google, comparten esta preocupación y tienen programas para acercar la informática a través de las universidades a los alumnos de secundaria, como el CS4HS (Computer Science for High School) en el que participa la Universidad Rey Juan Carlos en España.

En este artículo se pretenden analizar las razones que han llevado a esta situación desde el punto de vista de los jóvenes y los centros de educación superior, y una perspectiva de las distintas iniciativas innovadoras que se están llevando a cabo por parte de las Universidades y Administraciones públicas para acercar la realidad de la profesión informática a toda la sociedad y contrarrestar esta tendencia.

\section{2.- POSIBLES CAUSAS DEL DESCENSO DE LA MATRICULACIÓN EN TITULACIONES TIC}

Mucho se ha especulado en relación al descenso de la matriculación en las ingenierías y titulaciones relacionadas con las TIC, con mayor o menor acierto. Sin la intención de hacer un estudio pormenorizado de la extensa bibliografía que se ha publicado alrededor de este tema -en especial en países anglosajones-, incidiremos en las causas que más repiten los expertos procurando dar una perspectiva crítica a las mismas.

Probablemente la razón más argumentada es la falta de información acerca de la realidad profesional de los ingenieros TIC cuando los alumnos de secundaria van a hacer la elección de su futura carrera y Universidad (O'Lander, 1996; Lomerson \& Pollacia, 2006; Lenox \& Woratschek, 2008). Quizá el momento crítico sea incluso antes, cuando han de decidir la rama de bachillerato en la que van a comenzar a especializarse, ya que esa opción condiciona las carreras que después podrán cursar.

Si bien es cierto que existe una falta de información, es discutible su influencia directa. Cernuda del Rio \& Riesco (2010) muestran que el comportamiento que hasta ahora había sido cíclico en las matriculaciones en informática no se corresponde con la disponibilidad de información, ya que en los años 80 las TIC eran grandes desconocidas para buena parte de la sociedad y el número de alumnos sobrepasaba las plazas universitarias en estas titulaciones. Desde entonces el conocimiento de las tecnologías de la información y su uso ha ido en aumento. Es más, resulta curioso que la primera caída de matriculación en las ingenierías TIC en EE.UU. se haya producido al mismo tiempo que se han hecho esfuerzos por incorporar ordenadores en los centros de

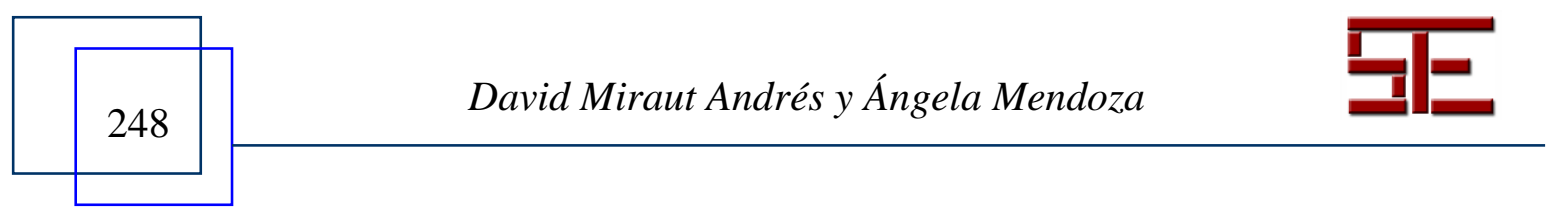




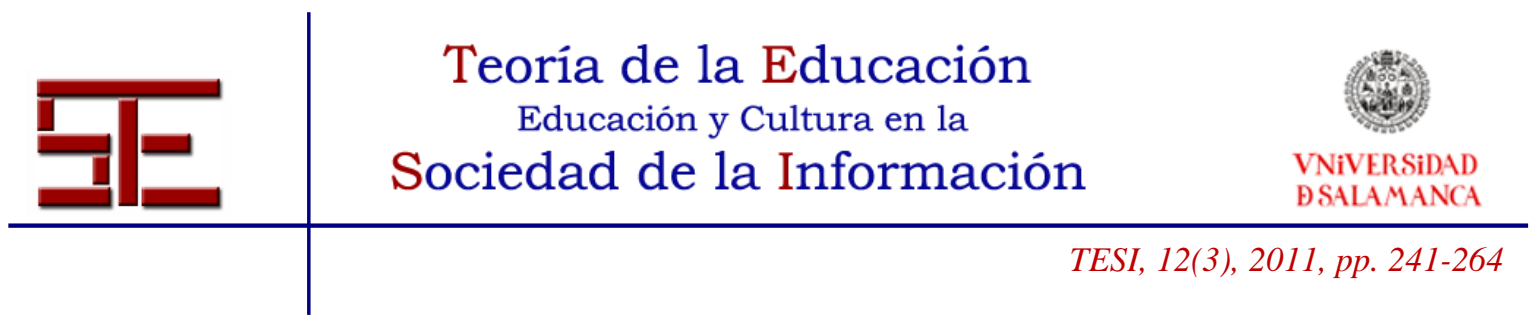

educación secundaria (O’Lander, 1996; Miraut, 2010), por lo que no lo podemos considerar el desconocimiento como el factor más influyente en la toma de decisión de los alumnos.

Probablemente no es tanto la falta de información como la calidad de la misma el factor que más influye (Carter, 2006). A menudo, la gente joven tiene una percepción errónea de la labor de los ingenieros informáticos y de telecomunicaciones, que es reforzada por el estereotipo que se presenta en películas y series de televisión. Por un lado, se presentan personas inteligentes, fascinadas por la tecnología y altamente capacitadas para interactuar con ella (López et al., 2010); pero el desarrollo de esas habilidades ha sido resultado de un gran esfuerzo y sacrificio, trabajando hasta horas intempestivas en labores tediosas y aburridas, de forma aislada, hasta el punto de convertirse en seres asociales, con problemas de comunicación, y numerosas excentricidades que dan lugar a una imagen de los informáticos que raya lo "friki" (Frieze, 2005). Rara vez se presenta en los medios la realidad del trabajo de los ingenieros, en grandes y medianos equipos, con diferentes especialidades, en los que la comunicación es primordial. Y las figuras mediáticas suelen estar más ligadas a las iniciativas empresariales -como Bill Gates o Steve Jobs- que por sus obras, como suele suceder en otras profesiones.

La facilidad para compartir y reutilizar partes código ha posibilitado que los ingenieros informáticos no tengan que enfrentarse a las limitaciones físicas con las que nos encontramos en otras disciplinas cuando los proyectos alcanzan cierta envergadura. Gracias a este factor y a la mejora continua de las prestaciones de los procesadores de propósito general, la complejidad del software ha crecido exponencialmente en las últimas décadas a la vez que lo hace la facilidad con la que podemos trabajar con componentes de alto nivel. A menudo se compara el diseño y construcción de sistemas operativos y procesadores con el de grandes maravillas de la humanidad como las pirámides de Egipto, por su complejidad y el esfuerzo coordinado de la gran cantidad de ingenieros que los han hecho posible. Sin embargo, rara vez se presentan como tales en los medios de comunicación, pues aunque los resultados son indudablemente prácticos, la obra no puede apreciarse en imágenes por lo abstracto de su naturaleza.

Una década después de la caída de las empresas "punto com", su eco todavía permanece, dejando tras de sí una imagen de cierta volatilidad en las empresas tecnológicas, hasta el punto de que muchos jóvenes perciben este tipo empleo como puestos en los que los ciclos de oferta y demanda se alternan en una sucesión en la que cada vez hay menos donde elegir (Lenox \& Woratschek, 2008). La realidad no podría

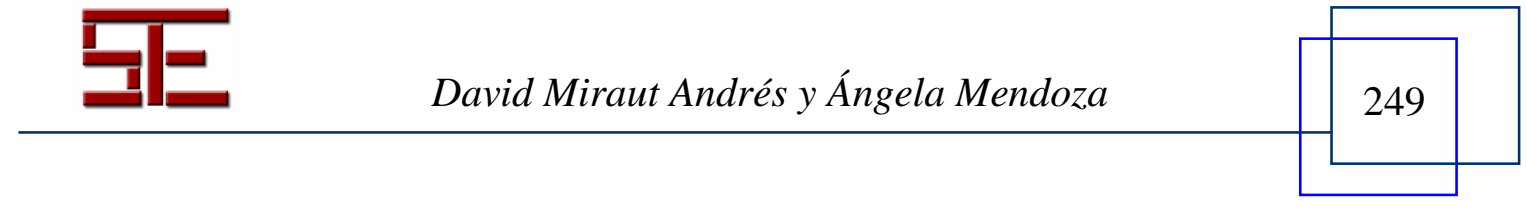




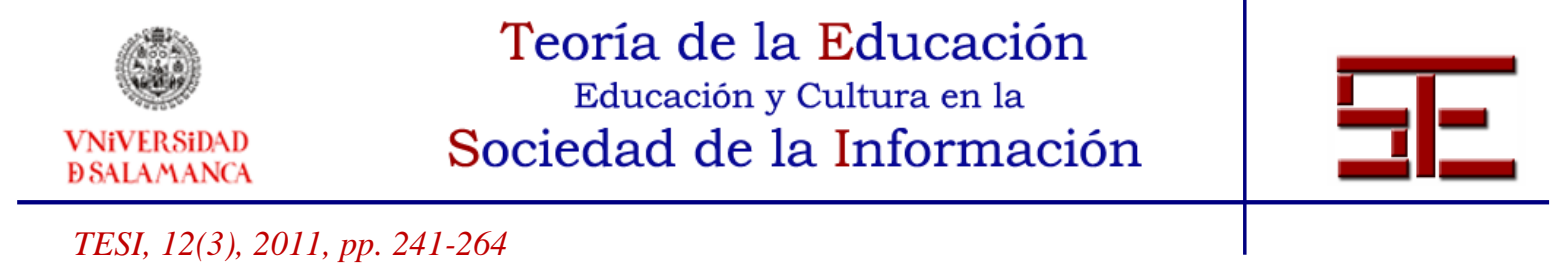

ser más distinta, las empresas han aprendido mucho del despliegue de Internet y las altas expectativas que a corto plazo tenían los inversores, esa experiencia ha consolidado un mercado mucho más diverso y robusto que el anterior, basado principalmente en servicios en nuestro país, con una demanda de nuevos ingenieros que crece a un ritmo mucho mayor que el de cualquier otra ingeniería, como ya se ha comentado (Patterson, 2005). Por ello, este temor es completamente infundado, al igual que el de salarios bajos o condiciones precarias de trabajo.

La falta de ingenieros para satisfacer la demanda en países occidentales ha forzado a las empresas a externalizar parte de sus desarrollos para no dejar de ser competitivas. Según los estudios publicados, este hecho preocupa especialmente a los alumnos estadounidenses, al pensar que su remuneración puede estar en peligro al rivalizar con profesionales de países de economías emergentes con un sueldo mucho más bajo que la media estadounidense. Lo cierto es que las empresas tecnológicas son muy reticentes a externalizar las partes del negocio que suponen una ventaja competitiva y la externalización (outsourcing) no es sencilla dada la complejidad de las relaciones entre los distintos componentes software y los equipos que los desarrollan. En el año 2004, Bill Gates hizo un tour por las universidades más prestigiosas con el objetivo de desterrar esta idea de las mentes de los estudiantes de informática, asegurando que los empleos estratégicos no saldrían del país (Denning \& McGettrick, 2005). La situación en España es doblemente curiosa, por un lado, la buena relación y el idioma común con los países latinoamericanos ha llevado a muchas empresas a externalizar una parte de su desarrollo y, por otro, el tiempo ha demostrado que la demanda es tan grande que incluso ingenieros de titulaciones que no son afines son contratados para labores de análisis, diseño y programación en nuestro país. Sin embargo, el alto nivel de cualificación de los ingenieros TIC españoles -debido en parte a la exigencia en los planes de estudio que se describirá más adelante- los hace especialmente atractivos no sólo para el mercado interno, sino también para el internacional, donde son muy bien valorados, por lo que la proactividad, el talento y la especialización se pagan muy bien. La reciente fundación del Colegio de Ingenieros Informáticos a nivel nacional tiene como uno de sus objetivos defender que esta situación se siga manteniendo así ante instituciones y empresas.

Numerosos investigadores ponen de manifiesto que parte de la falta de información de los alumnos de secundaria es consecuencia directa de la falta de una formación homogénea en los profesores de las asignaturas de Tecnología e Informática en los centros de educación secundaria. En parte debido a la fuerte demanda laboral

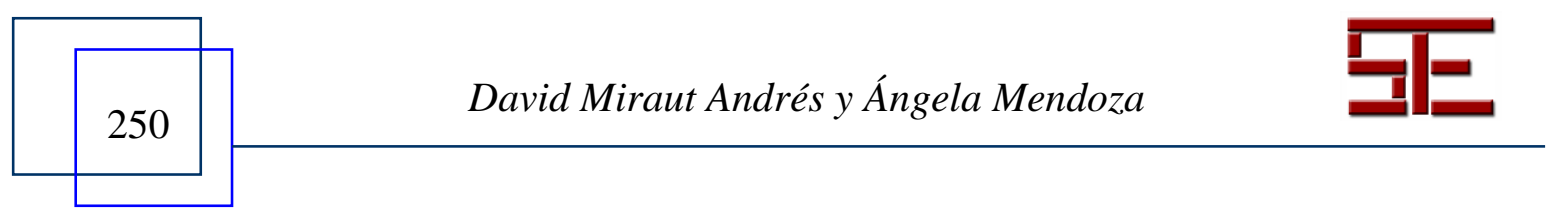




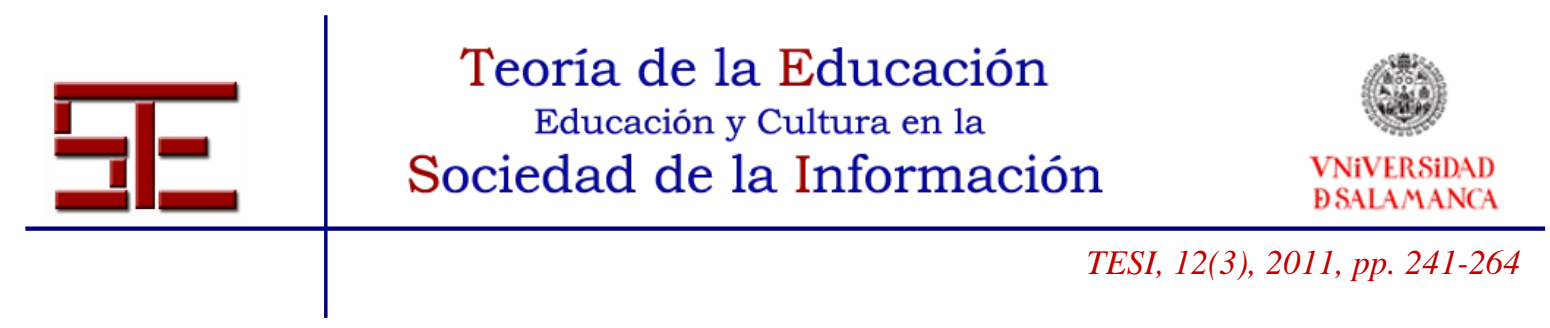

mencionada anteriormente, es relativamente raro encontrar casos de profesores de instituto informáticos. La falta de formación de profesorado lleva muchas veces a que la clase de informática tenga un contenido principalmente ofimático, en lugar de impartir conocimientos que den a los alumnos una perspectiva real y práctica de la disciplina que se están estudiando (Ali \& Shubra, 2010). Por ello desde las asociaciones profesionales se ha pedido a nivel internacional y con especial fuerza en EEUU que se regule al igual que se hace con otras especialidades en la enseñanza de secundaria (O'Lander, 1996; Patterson, 2005). Desde el punto de vista de los autores, es responsabilidad de las universidades crear contenidos, problemas atractivos, ideas de proyectos y otros tipos de material y cursos que puedan facilitar la tarea a los profesores de instituto y desarrollen las capacidades analíticas y de pensamiento abstracto de los estudiantes, de modo que tengan una amplia variedad donde elegir y puedan hacer más entretenidas y rigurosas sus clases (Miraut et al., 2010).

A menudo los alumnos de secundaria tienen un concepto de los estudios y la profesión informática muy ligados al concepto de la escritura de código. Para un ingeniero informático la programación tiene un sentido más amplio que abarca el diseño, desarrollo, testeo, depuración, documentación y mantenimiento del software; que no puede separarse del análisis de algoritmos y la teoría de la complejidad. El corazón y el alma de la informática se basa en 5 pilares interrelacionados: el pensamiento de sistemas, el modelado/diseño de los mismos, la programación, la arquitectura (de computadores) y la innovación (Denning \& McGettrick, 2005).

La innovación no es un don de unos pocos, sino que es la clave del papel de los profesionales TIC. No es una cuestión de suerte o de un talento especial; es una habilidad que se adquiere con la práctica (Drucker, 1985). La innovación puede ser aprendida, y los planes de estudio en informática suelen alentar su espíritu desde el primer curso hasta el último, en el que se realiza el proyecto de fin de grado. Para que resulte atractivo a los alumnos, este aprendizaje debe ser práctico y continuo, de modo que acaben por verlo como algo natural, fomentando su creatividad, el desarrollo del pensamiento crítico y las capacidades de análisis. La búsqueda de oportunidades para la innovación se acaba por convertir en un hábito a lo largo de los cursos, donde se preparan para un mercado en permanente cambio.

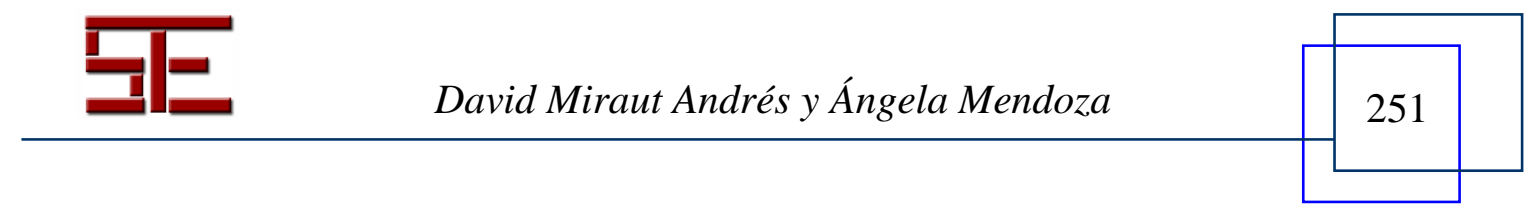




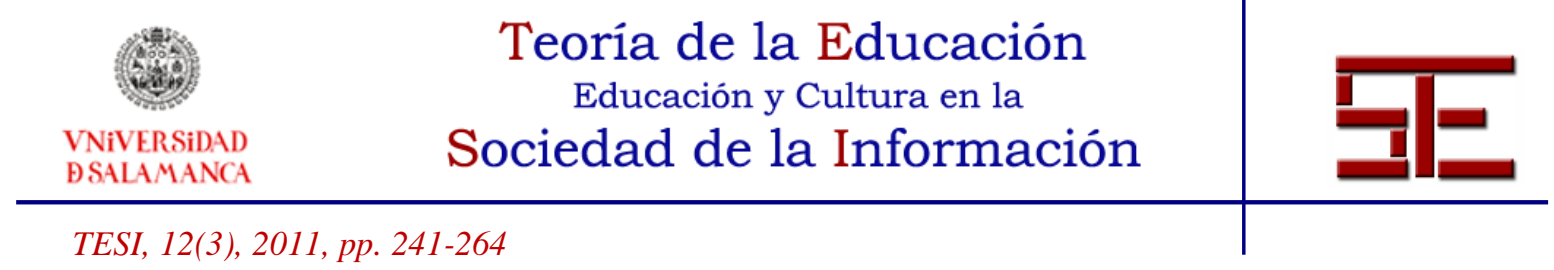

La creatividad y la innovación están presentes en cada aspecto del trabajo de los profesionales TIC cuando buscan nuevas soluciones. A diferencia de otros ingenieros, no necesitan grandes instalaciones para trabajar, ni están limitados por las leyes de la física en el sentido habitual. Los ingenieros de computadores sólo necesitan como herramienta un ordenador; todo cuanto imaginen puede ser creado virtualmente en él. Pero esta libertad creativa no estimula a los jóvenes, porque en la mayor parte de los casos no han tenido la oportunidad de experimentarla.

A pesar de su reducido porcentaje, la presencia en la industria de las mujeres es muy apreciada, ya que los miembros de este colectivo han dado continuas muestras de una excepcional capacidad en este campo, y destacan por su iniciativa, creatividad, liderazgo y confianza en sus puestos de trabajo (Roberts et al, 1992). La dificultad de llegar a una masa crítica de mujeres en las clases universitarias es una de las principales barreras señaladas por los expertos, ya que dificulta que se sientan parte de la comunidad aunque se relacionen fluidamente con el resto de colectivos. Esta situación no es diferente en otras carreras técnicas en la actualidad que tienen asociado un estereotipo fuertemente masculino, ni tampoco a la situación que se vivía hace unos años en Medicina, Arquitectura o Derecho. En estas últimas, las mujeres han sabido romper la barrera venciendo una oposición muy fuerte, y la situación se ha invertido. En comparación, las titulaciones TIC son mucho más jóvenes y no se puede argumentar que tengan una tradición de profesionales históricamente masculina (Cernuda del Rio \& Riesco, 2010).

Desde el punto de vista de los autores, la causa principal del descenso de las matriculaciones es consecuencia de los pasos que dieron las instituciones académicas desde mediados de los años 80 hasta mediados de los años 90 para reducir el número de alumnos en las titulaciones TIC. En aquella época, el número de universidades en España -y también en EE.UU. - era menor y la demanda era tan grande que no podía atenderse con suficiente calidad. Con el objetivo de aliviar las clases masificadas, muchas universidades optaron por imponer criterios de selección más estrictos (Roberts et al, 1992), se aumentó el nivel de exigencia en las asignaturas y se modificaron los planes de estudios para convertir los primeros cursos en filtros para limitar la entrada en el campo de las TIC. Estas medidas tuvieron un efecto a largo plazo desproporcionadamente negativo en la matriculación de las mujeres y otras minorías, reforzado por las causas antes mencionadas.

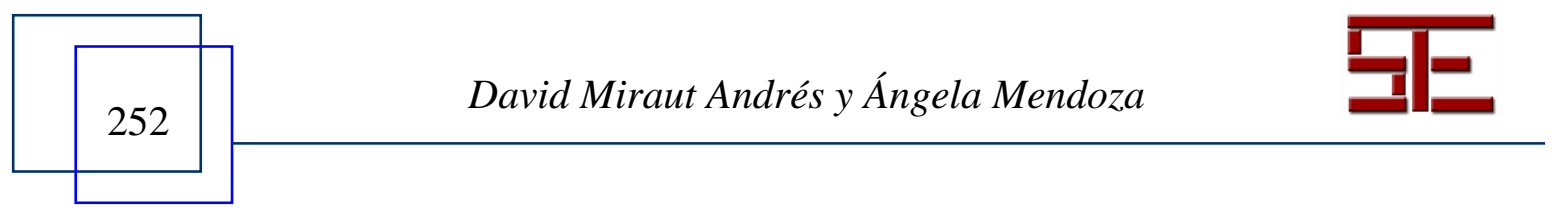




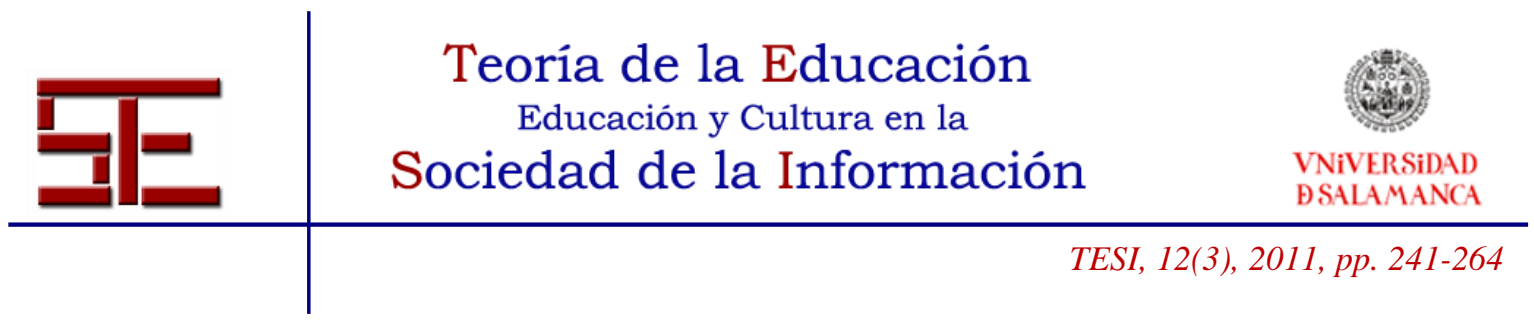

La situación actual en España es radicalmente diferente, se han creado nuevas universidades y el número de profesores en las titulaciones TIC ha crecido considerablemente para satisfacer las necesidades de la industria, sin embargo, la herencia de un pasado reciente sigue haciendo mella y pone en peligro el futuro inmediato de nuestra industria. Por ello es necesario que todos los agentes tomen medidas para cambiar esta situación.

\section{3.- INICIATIVAS EDUCATIVAS}

Podemos distinguir tres niveles en las iniciativas educativas en cuanto a su alcance y el tipo de medios que se han utilizado para ponerlas en marcha: internacionales (entre las que se encuentran las iniciativas basadas en software), nacionales y locales. Todas ellas tienen por objetivo mejorar la percepción de la sociedad acerca de las profesiones TIC, involucrando activamente a los más jóvenes en actividades tecnológicas con el objetivo de despertar vocaciones en este campo, en especial en el colectivo de las mujeres.

\section{1.- Iniciativas a nivel global}

Las empresas de tecnología no suelen distinguir entre fronteras a la hora de buscar talento y profesionales, siendo el inglés la lengua común en las TIC. Por ello tampoco hacen diferencias a la hora de proponer proyectos que acerquen a los jóvenes a la informática.

Los ejemplos son muchos y cada vez más numerosos. Probablemente una de las iniciativas más loables es la que abandera Google: Computer Science for High School, en la que se trata de promocionar la informática y las habilidades relacionadas con el pensamiento computacional en los colegios y escuelas de secundaria. A través de universidades se celebran cursos de verano y campamentos urbanos de 2 a 3 días en los que se trata de incentivar la curiosidad de los jóvenes. Entre las universidades participantes se encuentran el Instituto Tecnológico de Massachusetts (MIT), la Universidad Carnegie Mellon, la Universidad de California, la Universidad de Illinois, la Universidad de Texas, la Universidad de Washington, el University College de Dublín, el ETH de Zúrich, la Universidad Manchester y la Universidad Rey Juan Carlos en España.

En estos cursos de verano se realizan talleres, ponencias y actividades dirigidas a alumnos y a profesores de informática de centros de educación secundaria, que les ayudan a conocer mejor el mundo de la informática de forma divertida y rigurosa.

El material creado en los cursos se pone a libre disposición de la comunidad para que pueda ser reaprovechado en los institutos, de modo que los profesores puedan

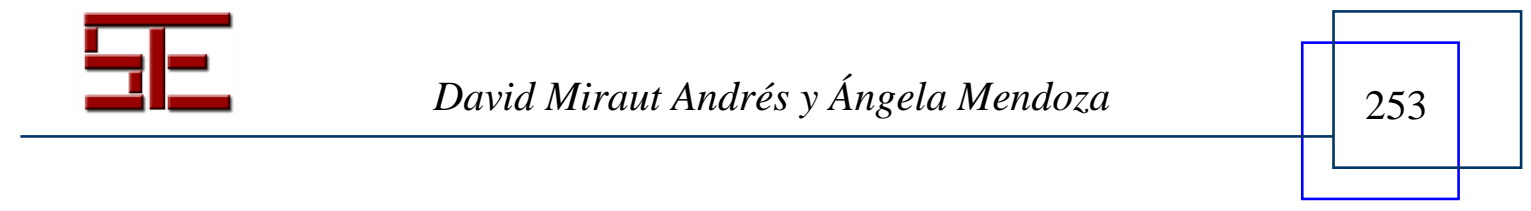




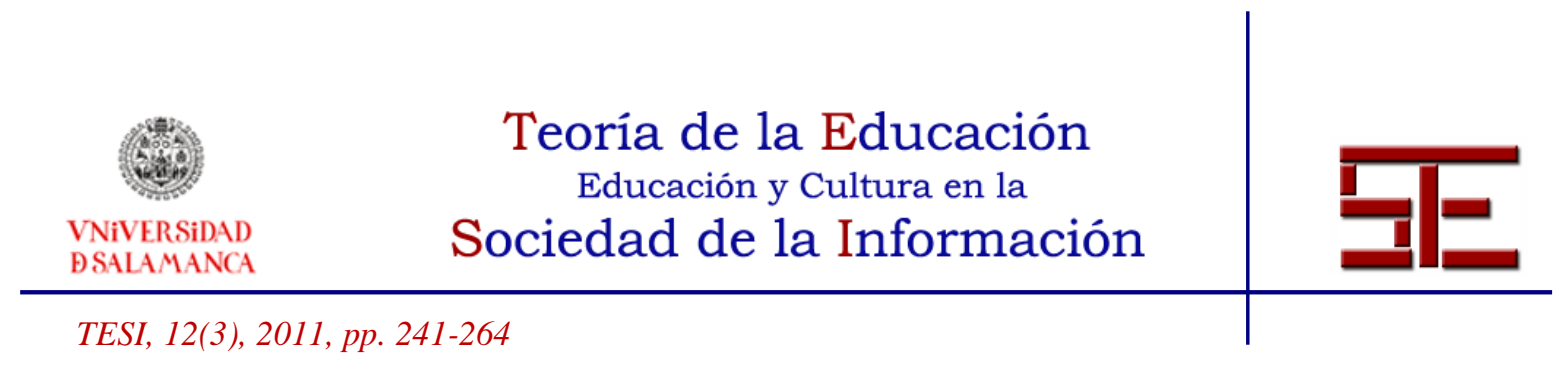

reproducir las actividades con sus grupos aunque no hayan tenido la oportunidad de asistir.

Las asociaciones profesionales de informática -como el ACM (Association for Computing Machinery) - tienen secciones que están centradas en esta problemática y organizan actividades con el mismo objetivo. Algunas de las más populares son los certámenes de programación, que se han multiplicado en los últimos años gracias a la ubicuidad de la red. Entre las tradicionales destacan la Competición Internacional ACM de Programación y la Olimpiada Internacional de Informática, centradas en la resolución de problemas y el diseño de algoritmos. Otras variantes como los certámenes de animación han tenido también una gran aceptación por mezclar habilidades informáticas con el arte.

Las asociaciones profesionales de mujeres ingenieras, tales como el IEEE WIE (IEEE Women in Engineering), CWIT (The Center for Women \& Information Technology) y AMIT (Asociación de Mujeres Investigadoras y Tecnólogas) llevan a cabo numerosas actividades para animar a las jóvenes y canalizan las donaciones para proveer de becas a este colectivo. Su labor es muy destacable, porque el tipo de iniciativas que promueven son especialmente inspiradoras. Un ejemplo es la aparición estas navidades de un nuevo modelo de Barbie ingeniera informática a petición de estas asociaciones, que han asesorado a Mattel en su aspecto y accesorios con el objetivo de que las niñas puedan imaginarse un futuro en que como ingenieras puedan tener un impacto positivo en la vida diaria de las personas.

\section{2.- Iniciativas basadas en software}

Dado que el tipo de eventos de la sección anterior tiene una capacidad limitada, los equipos de las universidades y centros de investigación han aunado esfuerzos para crear soluciones software que pretenden dar respuesta a las necesidades de formación en los institutos en el campo de la informática, proveyendo material y documentación atractiva y gratuita que pueda ser utilizada de forma global.

El desarrollo de habilidades informáticas que lleven a los jóvenes de una posición pasiva como usuarios a una posición activa como creadores es fundamental para abrir su mente a las posibilidades que les brindan los ordenadores. El aprendizaje de un lenguaje de programación es una tarea ardua para la mayoría de los estudiantes, que se encuentran con muchas dificultades al comienzo, como por ejemplo la rigidez de la

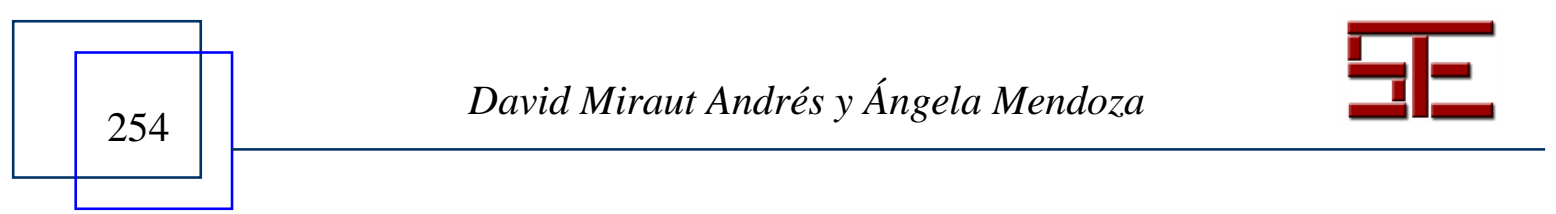




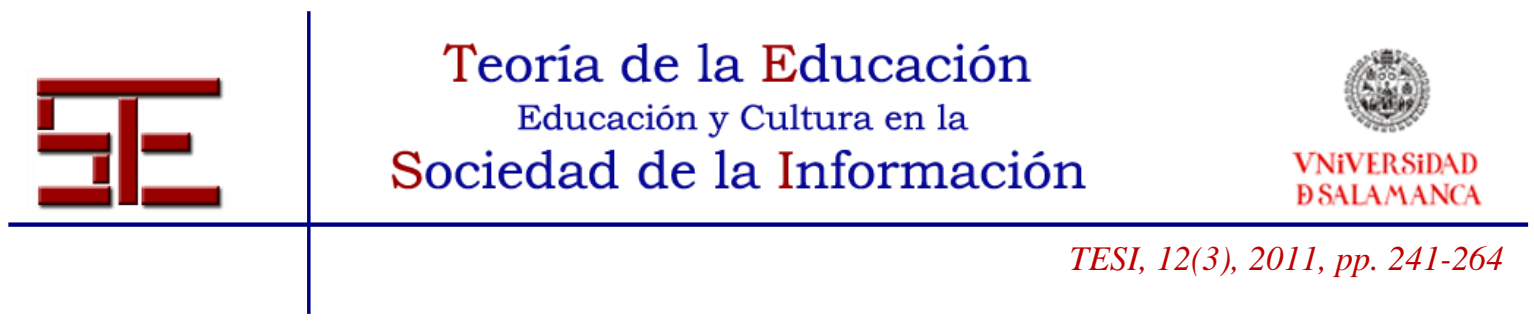

sintaxis, las estructuras que no les son familiares y la cantidad de esfuerzo invertido para comenzar a obtener resultados por pantalla.

A continuación se resumen algunas de las herramientas -ordenadas por grado de dificultad y edad a las que serían apropiadas- que pueden ser utilizadas para aliviar estos escollos en educación primaria y secundaria, de modo que los profesores puedan centrarse en conceptos más atractivos como la resolución de problemas, el diseño de algoritmos y el propio proceso de la creación de los programas:

\subsubsection{Kodu Game Lab}

$K o d u$ es una aplicación visual desarrollada por Microsoft, en la que es posible diseñar juegos 3D de forma intuitiva y sencilla, de manera que jóvenes entre 9 y 14 años puedan expresar su creatividad a través de la programación de forma completamente visual. Funciona en plataformas Microsoft Windows (XP, Vista y 7) así como en la XBoX 360. La iniciativa comenzó como una prueba de concepto por parte de un investigador de Microsoft con el objetivo de que su hija desarrollase habilidades de pensamiento computacional a través del desarrollo de juegos 3D. Tras un estudio en colaboración con la Universidad de Barbara en el que se evaluó la influencia de los juegos de ordenador en el aprendizaje en niños a partir de 5 años en ciencia y tecnología, comprobaron que este tipo de herramientas les ayuda a captar los conceptos más rápidamente, pero que deben tener libertad de experimentación para profundizar en conceptos avanzados. Así fue evolucionando Kodu hasta el producto que se ofrece gratuitamente. Los usuarios interactúan con elementos básicos, creando escenarios y personajes con distintas habilidades con los que interaccionan.

\subsubsection{Gamestart Mechanic}

Gamestart Mechanic es un entorno online de aprendizaje basado en juegos desarrollado por GameLab en la universidad de Wisconsin-Madison. El objetivo de esta aplicación es desarrollar juegos para el aprendizaje de diversas disciplinas a través de una interfaz visual que engancha a los alumnos. El sistema está pensado de manera que el juego puede ser guiado por profesores y padres que pueden interactuar y hacer un seguimiento de los progresos desde otros ordenadores.

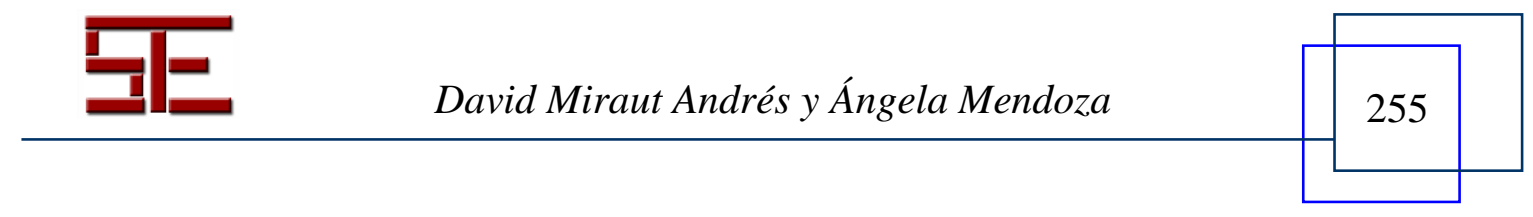




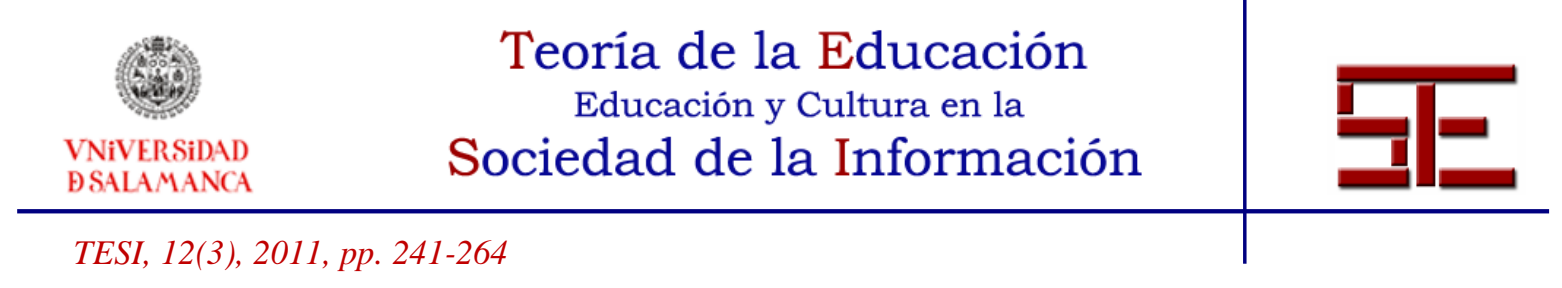

\subsubsection{Scratch}

Scratch es un programa desarrollado en el 2007 por LinfeLong Kindergarten Group en el MIT Media Lab. Con esta herramienta los niños, entre 6 y 15 años, pueden crear sus propias historias interactivas, juegos y música, y a través de ellos introducirse en los conceptos de programación. Se encuentra disponible gratuitamente en castellano para sistemas operativos Windows, MacOSX y Linux.

El nombre proviene de la técnica de scratching de los DJs, en la que suelen reutilizar piezas. En Scratch todos los objetos, gráficos, sonidos y secuencias de comandos pueden ser importados con facilidad a un nuevo programa para ser combinados de otras formas y así obtener resultados rápidos que motiven a los estudiantes.

La codificación se realiza a través de una interfaz muy intuitiva mediante el sistema de arrastrar y soltar bloques de código.

\subsubsection{Squeak}

Squeak va más allá de la metáfora de la creación de historias y propone una interfaz gráfica en la que se pueden combinar múltiples elementos multimedia (texto, video, sonido, música, gráficos $2 \mathrm{D}$, gráficos $3 \mathrm{D}$, TextToSpeech, etc). Así al programar se manipula el entorno mismo. La flexibilidad del sistema es muy grande y su lenguaje de programación orientado a objetos está basado en Smalltalk, de modo que cada uno de los objetos tiene su propia memoria y capacidad de comunicarse con mensajes con los demás.

Combinado con el entorno eToys se convierte en una potente herramienta de aprendizaje constructivista.

\subsubsection{Rapunsel}

Rapunsel es un juego de baile diseñado para enseñar a programar a niños de entre $10 \mathrm{y}$ 12 años. Está especialmente dirigido a las chicas, a las que se invita a explorar la programación mediante un conjunto de retos de dificultad gradual en un mundo tridimensional con gráficos muy llamativos, gracias al motor Torque Game Engine (TGE). El proyecto ha sido desarrollado por Universidad de Nueva York con la colaboración de empresas como Intel y Microsoft. Los estudios publicados (Plass, 2009) muestran que el diseño de la aplicación ayuda a romper los estereotipos y aumenta la motivación en el aprendizaje de numerosas disciplinas con una mezcla de competición y cooperación en los participantes.

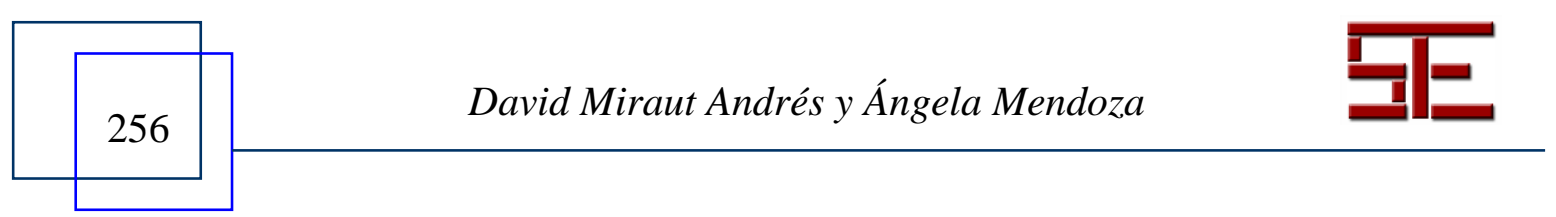




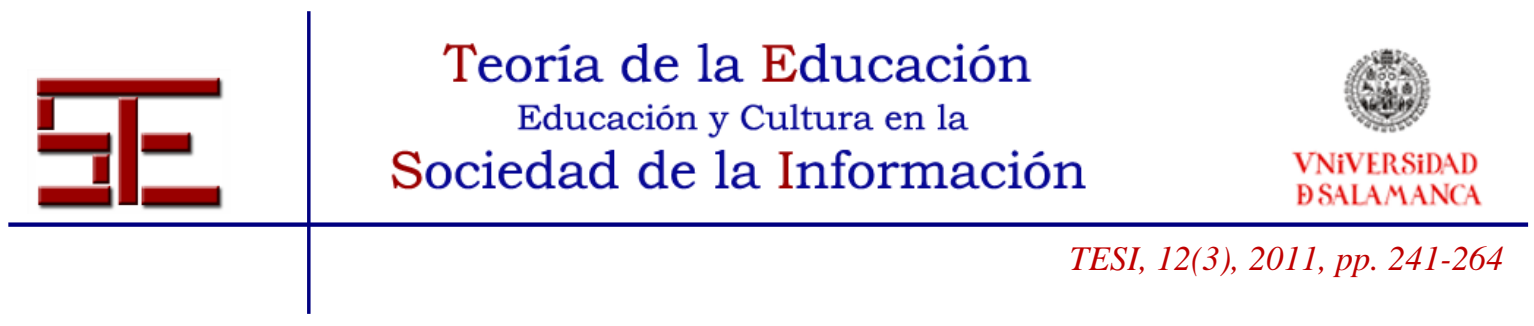

El software no está todavía disponible públicamente, pero se puede contactar con los autores.

\subsubsection{Greenfoot}

Greenfoot es un entorno de desarrollo visual e interactivo basado en el sistema BlueJ, para introducir conceptos básicos de programación orientada a objetos en jóvenes de entre 13 y 18 años. Está siendo desarrollado y mantenido por la Universidad de Kent y la Universidad La Trobe, con apoyo de Oracle. Los alumnos pueden desarrollar proyectos interactivos $2 \mathrm{D}$ y realizar pequeñas simulaciones físicas y experimentos científicos de forma visual. La interfaz es muy intuitiva, aunque, al igual que en los programas vistos anteriormente, su simplicidad y las limitaciones a la hora de exponer a los usuarios al código dificultan su progreso al llegar a un determinado nivel. Está disponible de forma gratuita en castellano para gran cantidad de sistemas operativos, al estar escrito en Java.

\subsubsection{AgentSheets}

AgentSheets es una aplicación visual desarrollada en 1996 por Alexander Repenning en la universidad de Colorado como parte de un proyecto de investigación interacción hombre-máquina. Permite el diseño y la creación de juegos de simulación web basados en agentes mediante una interfaz de arrastrar y soltar. Es lo suficientemente sofisticado como para poder realizar juegos del estilo a los Sims con inteligencia artificial. Para dar el paso de la interfaz visual que tiene el programa a un formato más tradicional el sistema puede traducir los proyectos a código fuente en Java.

Aunque sus capacidades de visualización están limitadas a gráficos $2 \mathrm{D}$, como en buena parte de los proyectos presentados, la facilidad con la que se pueden configurar los comportamientos de los agentes es un atractivo especial para los jóvenes, en especial las chicas.

Esta herramienta puede ser utilizada no sólo para juegos, sino para crear proyectos que desarrollen otras habilidades de pensamiento computacional en el contexto de todo tipo de asignaturas: Historia, Biología... gracias a la capacidad que tienen los agentes para comunicarse se pueden desarrollar simulaciones que visualicen muchos tipos de procesos de distinta naturaleza.

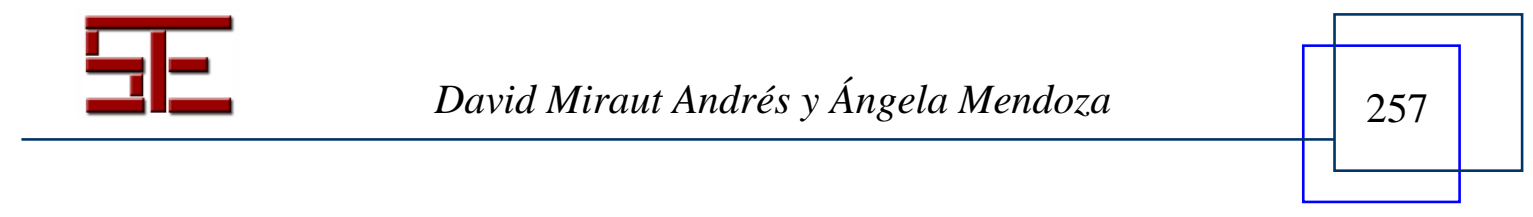




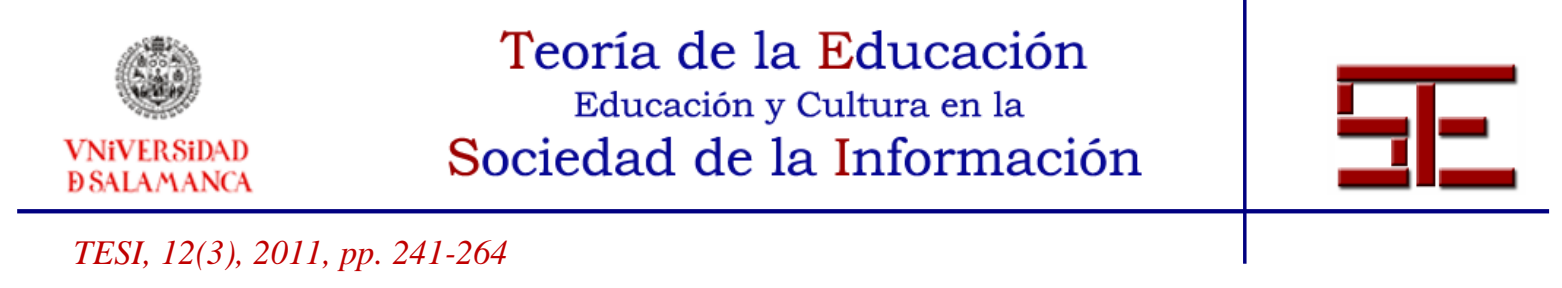

\subsubsection{Rebeca a través del espejo y Alice}

Rebeca y Alice son dos propuestas de innovación educativa que tratan de acercar el mundo de la programación de forma atractiva a los jóvenes, y muy especialmente a las chicas. A través de un entorno de programación 3D pueden crear, de forma sencilla, animaciones para contar historias, videojuegos interactivos y vídeos para compartir en Internet.

Alice fue desarrollado inicialmente como parte de un proyecto de investigación de Realidad Virtual del grupo de gráficos de Randy Pausch en la Universidad Carnegie Mellon. Este proyecto ha ido creciendo durante más de 15 años (Pausch et al, 1995; Kelleher, 2005, 2007, 2009), apoyado por numerosas facultades, profesores y alumnos. Rebeca es un software basado en Alice, desarrollado en la Universidad Rey Juan Carlos, en el que se han tratado de superar los principales escollos a los que se enfrentaban los estudiantes españoles en su uso (Miraut et al., 2010).

Ambos sistemas están pensados para servir como primera toma de contacto a la programación orientada a objetos, permitiendo a los estudiantes aprender conceptos fundamentales de programación dentro de un contexto creativo en el que pueden dejar volar su imaginación. Así, adquieren poco a poco destrezas importantes en su desarrollo profesional:

- Descubren y practican nuevas formas de pensar, que no se limitan a buscar una sola solución al problema, sino que lo atacan desde varias perspectivas simultáneamente.

- Adquieren la capacidad para expresar los algoritmos resultantes en un lenguaje formal.

- El desarrollo de pensamiento abstracto para concebir y transmitir ideas complejas de forma simple, de modo que se descomponga un programa con lógica en componentes más básicos

- Un cierto sentido de apreciación de la elegancia que les ayude a distinguir entre todas las perspectivas aquellas que son más adecuadas para resolver los problemas bajo ciertas condiciones.

Los estudiantes ven inmediatamente cómo se ejecutan sus programas animados, comprendiendo intuitivamente la relación entre las sentencias de programación y el comportamiento de los objetos en sus animaciones. Mediante la manipulación de objetos en un mundo virtual ganan experiencia con todo tipo de estructuras típicas en un curso de introducción a la programación.

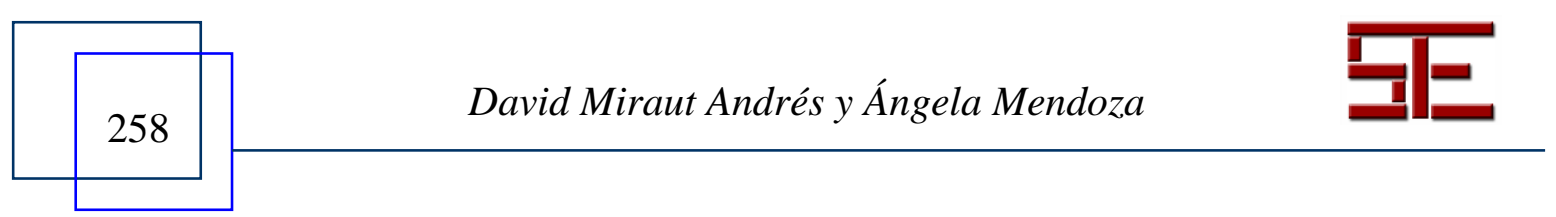




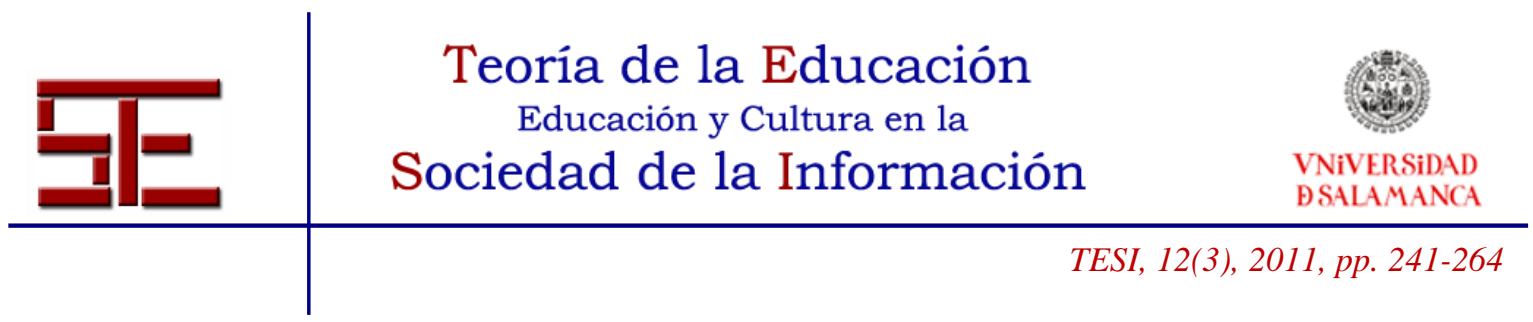

Gracias al uso de gráficos 3D, estas herramientas se comunican más eficientemente con una generación que ha crecido inmersa en el mundo visual de los videojuegos y películas de animación. Ambas herramientas demuestran que esto es posible sin perder rigor y profundidad en los contenidos que deben asimilar los alumnos.

Rebeca a través del espejo y Alice se pueden descargar para plataformas Windows y MacOSX. El equipo de desarrollo de Rebeca ha creado un conjunto de materiales didácticos que facilitan la tarea de profesores y alumnos al comenzar a utilizar la herramienta, y al igual que el programa se distribuye gratuitamente.

\subsubsection{DrJava}

DrJava es un entorno de programación tradicional desarrollado en Java diseñado para estudiantes que están dando sus primeros pasos en este lenguaje. Ha sido desarrollado por un equipo JavaPLT de la Universidad. La interfaz evalúa el código en tiempo real y tiene opciones que facilitan el desarrollo de aplicaciones multimedia.

\subsubsection{BlueJ}

BlueJ es un entorno de desarrollo en Java creado con objetivos educacionales que puede ser utilizado para el desarrollo de software a pequeña escala. La interfaz es muy intuitiva y representa gráficamente en la zona principal la estructura de clases de la aplicación que se está programando, que puede probarse interactivamente a medida que se crean nuevos objetos. De modo que visualmente se puede conocer el estado de los objetos y depurar el código. Junto con DrJava son las opciones menos abstractas y que más se acercan a la forma de proceder a la hora de programar en el mundo ingeneril.

\section{3.- Iniciativas a nivel nacional}

Los gobiernos comparten la preocupación de la industria y las universidades por la caída en la matrícula de las ingenierías TIC, por ello y con el objetivo de seguir siendo competitivos en todas las ramas de la industria que aprovechan estas tecnologías, han tomado iniciativas a nivel nacional en el ámbito de la educación. En España cabe destacar las relacionadas con el acercamiento de los jóvenes a las nuevas tecnologías y las que procuran protegerlos de su mal uso.

El programa Escuela 2.0 para la innovación y modernización de los sistemas de enseñanza es un programa que tiene como objetivo integrar las TIC en los centros

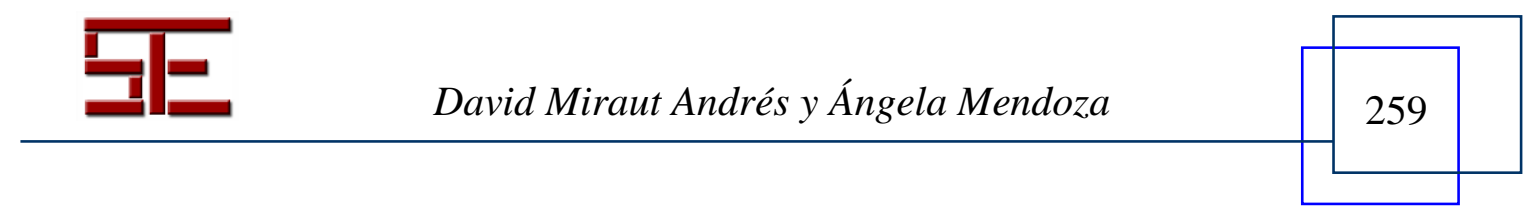




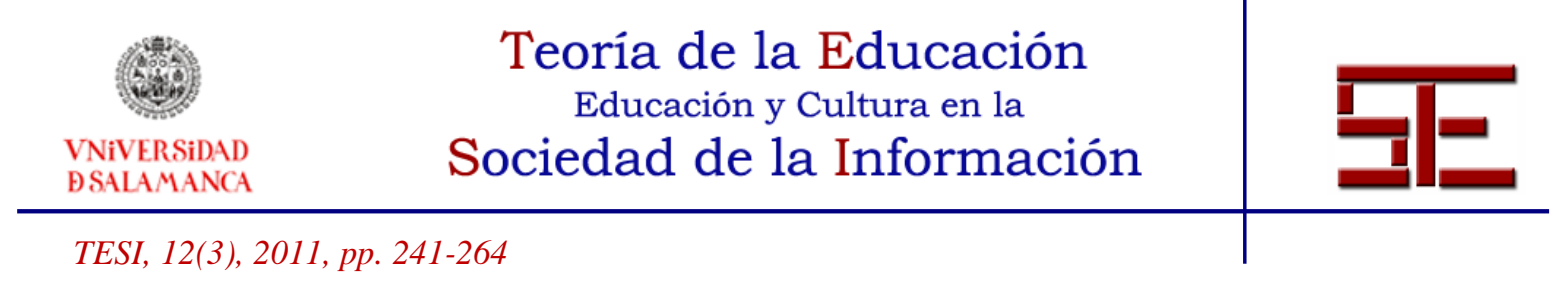

educativos públicos, de modo que estos recursos pasen a formar parte de los aspectos metodológicos y sociales de la práctica docente cotidiana (Miraut, 2010).

Las campañas de concienciación, como la de Pantallas Amigas, orientadas a sensibilizar sobre los riesgos de algunas prácticas inadecuadas que han surgido con el uso de las nuevas tecnologías.

Para que estas iniciativas tengan un efecto real en el problema considerado es necesario que los profesores aprovechen los nuevos medios para mostrar de forma atractiva la realidad profesional de las ingenierías TIC. Programas como Rebeca (Miraut et al, 2010) pueden ser una opción sugerente para los alumnos, que los motive a estudiar dentro y fuera del aula.

\section{4.- Iniciativas a nivel local}

Con la adopción del Espacio Europeo de Educación Superior muchas universidades han aprovechado para adaptar sus planes de estudio a los nuevos tiempos y se han creado nuevas especialidades de titulaciones TIC más atractivas para los estudiantes. Así han surgido numerosos grados en Multimedia (siguiendo el ejemplo de GeorgiaTech [Carter, 2006]), Diseño de Videojuegos (una de las industrias que mueve más dinero en nuestro país), Web (Schneiderman, 2007), titulaciones dobles (Lenox \& Woratschek, 2008) y multidisciplinares, cuya especialidad puede estar aún más valorada por la industria.

Las universidades ofrecen cursos cero (también llamados cursos puente) para reforzar la base de los estudiantes de primer año que consideren que pueden llegar a tener dificultades, para que no perciban las asignaturas de estas ingenierías como difíciles (Sloan \& Troy, 2008). En el caso de la informática las habilidades analíticas son especialmente importantes, y los alumnos que no tienen experiencia previa en programación se pueden beneficiar del uso de las iniciativas software descritas anteriormente con ayuda de sus profesores y la participación activa en las asociaciones de estudiantes.

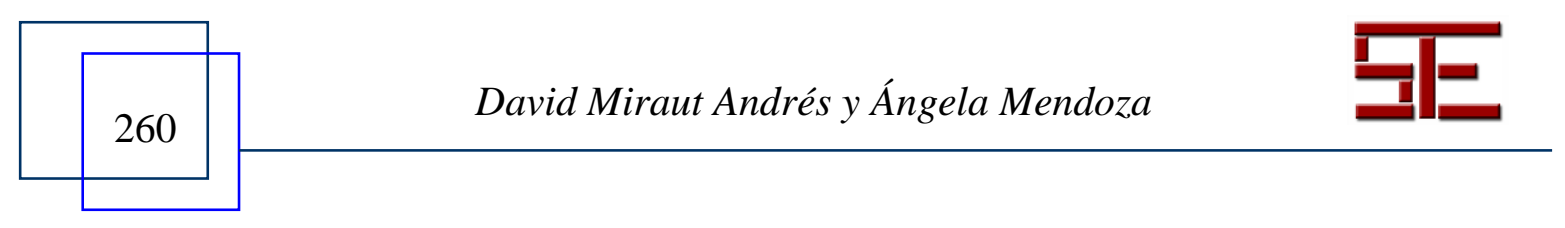




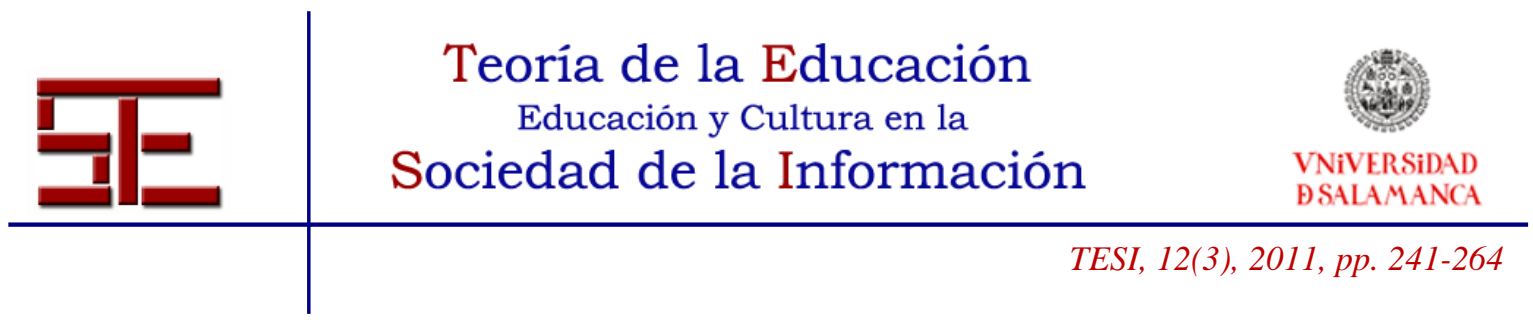

\section{4.- CONCLUSIONES}

Tal y como demuestran los estudios citados y a pesar de las iniciativas a nivel nacional, los estudiantes de países occidentales no suelen ser conscientes de la labor profesional de los ingenieros TIC más allá de los estereotipos que ven en películas y series de televisión. Tienen la percepción de que la carrera requiere un gran esfuerzo, en especial las asignaturas que en otro tiempo fueron comunes a todas las ingenierías en los primeros cursos. Esta percepción errónea les hace decantarse por otras titulaciones sin llegar a explorar su afinidad por las ingenierías TIC.

Para dar respuesta a las necesidades de la industria debe aumentar el número de estudiantes, especialmente mujeres, y para ello es necesario presentar la cara más práctica y humana de la informática. Sólo será posible si todos los agentes involucrados se implican, en especial aquellos que tienen un contacto directo con los jóvenes para ayudarles a encontrar su vocación.

En este artículo se ha procurado dar una perspectiva de las numerosas iniciativas que se están poniendo en marcha a distintos niveles, con especial hincapié en aquellas que pueden favorecer la incorporación de la mujer en las ingenierías TIC y las que pueden proporcionar herramientas y formación a sus formadores.

El proyecto Rebeca a través del espejo -mencionado en el artículo- ha sido financiado por la VI convocatoria de ayudas a la innovación y mejora de la docencia para el curso 2010/11 de la Universidad Rey Juan Carlos, y ha contado con el apoyo de la Escuela Técnica Superior de Ingeniería Informática en la organización y promoción de actividades para jóvenes estudiantes.

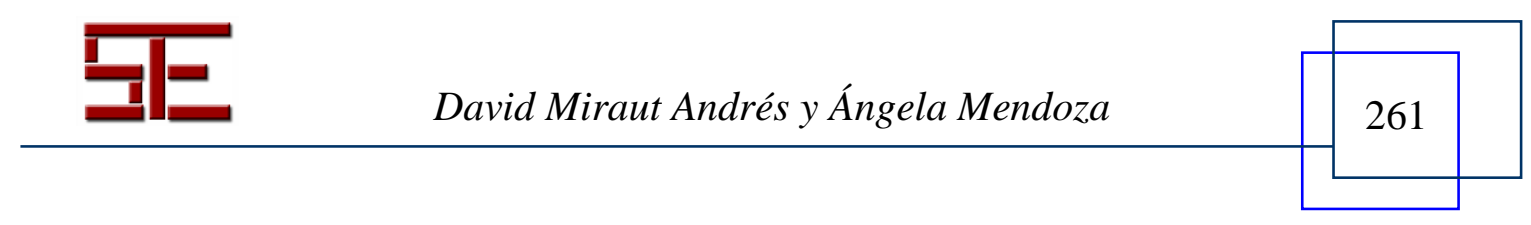




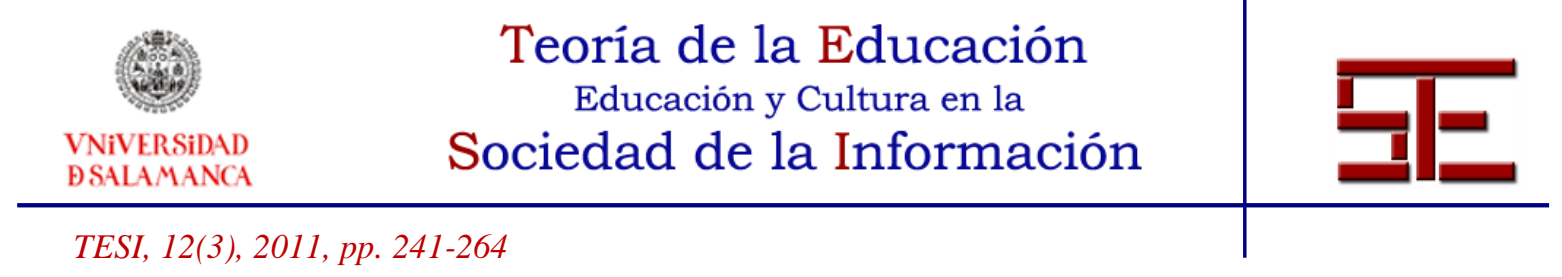

\section{BIBLIOGRAFÍA.}

Ali, A. y Shubra, C. (2010). Efforts to Reverse the Trend of Enrollment Decline in Computer Science Programs. The Journal of Issues in Informing Science and Information Technology, 7, 209-225.

Becerra-Fernandez, I., Elam, J. y Clemmons, S. (2010). Reversing the landslide in Computer related degree programs. Communications of the ACM, 53 (2),127-133.

Bureau (2010). Bureau of Labor Statistics (2010-11) U.S. Department of Labor, Career Guide to Industries, 2010-11 Edition, "Computer Systems Design and Related Services”, Extraído de Bureau of Labor Statistics web site el 20 de junio de 2010: http://www.bls.gov/oco/cg/cgs033.htm

Carter, L. (2006). Why students with an apparent aptitude for computer science don't choose to major in computer science. En SIGCSE '06: Proceedings of the 37th SIGCSE technical symposium on Computer science education (pp. 27-31). Houston, Texas, USA: ACM Press.

Cernuda del Río, A. y Riesco, M. (2010). ¿Investigamos correctamente sobre la baja matriculación de mujeres en Informática? En Actas de JENUI 2010 (pp. 257-264). Santiago de Compostela.

Denning, P. J. y McGettrick, A. (2005). Recentering computer science. Communications ACM, 48 (11), 15-19.

Drucker, P. (1985). Innovation and Entrepreneurship. Harper Business.

Frieze, C. (2005). Diversifying the images of computer science: undergraduate women take on the challenge! SIGCSE Bulletin, 37 (1), 397-400.

Graham, S. y Latulipe, C. (2003). CS girls rock: sparking interest in computer science and debunking the stereotypes. En SIGCSE '03: Proceedings of the 34th SIGCSE technical symposium on Computer science education (pp. 322-326). Reno, Nevada, USA: ACM Press.

INE (2010). Datos extraídos de la base de datos pública el 10 de abril de 2011.

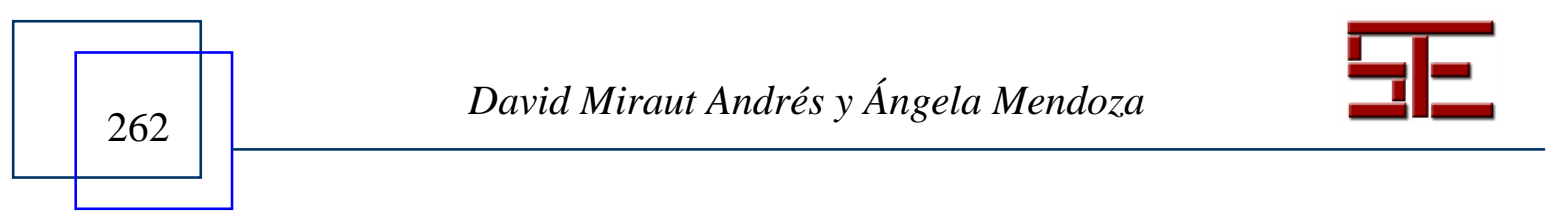




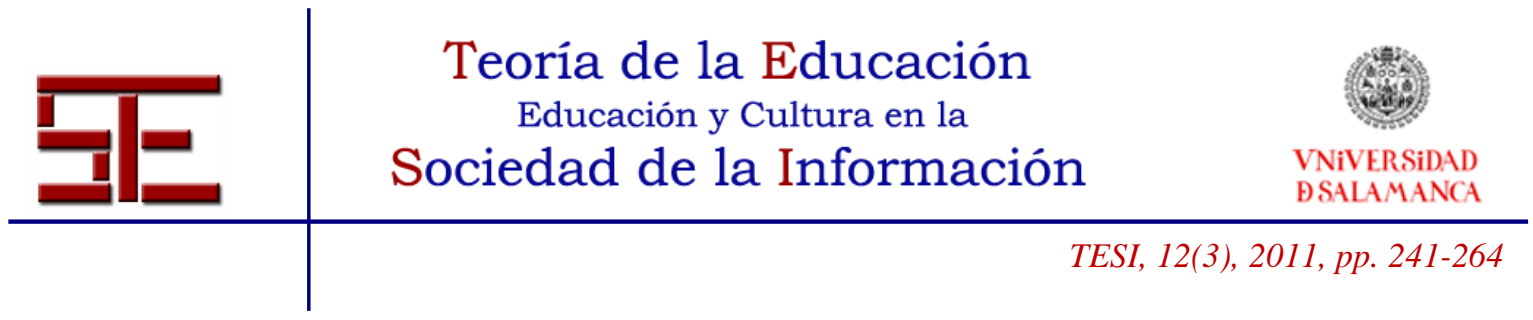

Jobs, S. (1980). Computers are like a bicicle for our minds. Entrevista incluida en Memory and Imagination. New Pathways to the Library of Congress. Accesible a través de http://www.youtube.com/watch?v=ob_GX50Za6c; extraído el 15 de abril de 2011.

Kelleher, C. (2009). Barriers to Programming Engagement. Advances in Gender and Education, 1 (1), 5-10.

Kelleher, C. y Pausch, R. (2005). Lowering the barriers to programming: A taxonomy of programming environments and languages for novice programmers. ACM Computing Surveys, 37 (2), 83-137.

- (2007). Using Storytelling to Motivate Programming. Communications of the $A C M, 50$ (7), 58-64.

Lenox, T. L. y Woratschek, C.R. (2008). Exploring Declining CS/IS/IT Enrollments. Information Systems Education Journal, 6 (44), 1-11.

Miraut, D. (2010). El sueño de Isaac y la transformación de los sistemas educativos en la sociedad de la información En Hernández Serrano M. J. y Fuentes Agustí, M. (Coords.) La red como recurso de información en educación. Revista Teoría de la Educación: Educación y Cultura en la Sociedad de la Información, 12 (1), 240-266.

Miraut, D., Mendoza, A., Mata, S. y Pastor, L. (2011). Rebeca through the looking glass: a 3D adventure to learn to program. En las actas del International Symposium on Distributed Computing and Artificial Intelligence, Advances in Intelligent and Soft Computing (pp. 225-232). Salamanca, España: Elsevier.

O'Lander, R. (1996). Factors effecting high school student's choice of computer science as a major. En CQL '96: Proceedings of the symposium on Computers and the quality of life (pp. 25-31). Philadelpia, Pennsylvania, USA: ACM Press.

Lommerson, W. L. y Pollacia, L. F (2006). Declining CIS Enrollment: an examination of pre-college factors. Information Systems Educational Journal, 4 (35), 1-13.

López, D., Cruz, J. L., Álvarez, C., Fernández, A. y Sánchez, F. (2010). Mamá, de mayor quiero ser ingeniero/a en informática. En Actas de JENUI 2010 (pp. 257-264). Santiago de Compostela.

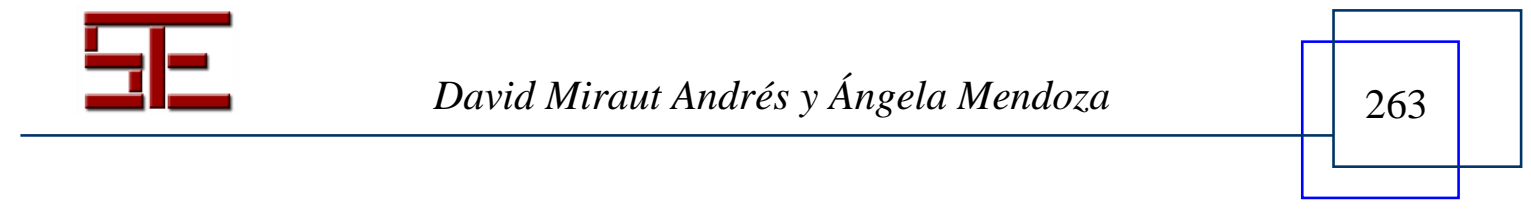




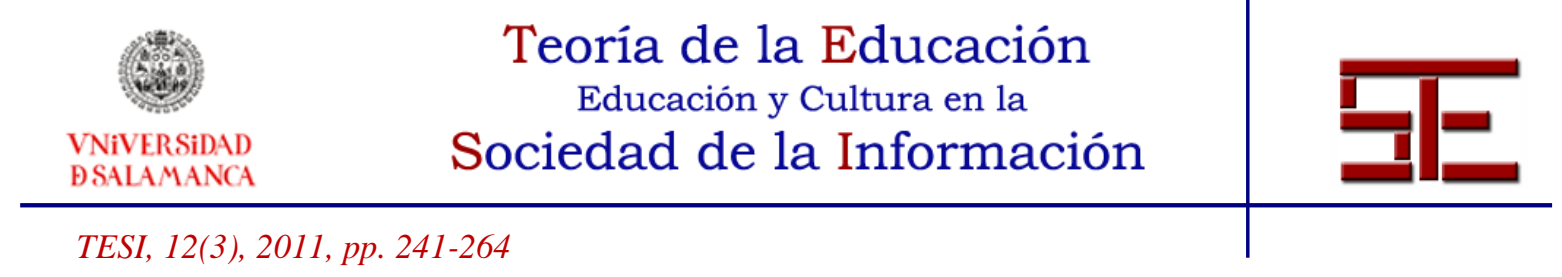

Patterson, D. A. (2005). Restoring the popularity of Computer Science. Communications ACM, 48 (9), 25-28.

Pausch, R., Burnette, T., Capeheart, A. C., Conway, M., Cosgrove, D., DeLine, R., Durbin, J., Gossweiler, R., Shuichi Koga y White, J. (1995). Alice: Rapid Prototyping System for Virtual Reality. IEEE Computer Graphics and Applications, 15 (3), 8-11.

Plass, Jan L., Goldman, R., Flanagan, M. \& Perlin, Ken. (2009). RAPUNSEL: Improving Self-Efficacy and Self-Esteem With an Educational Computer Game. En las actas de la 17th International Conference on Computers in Education (pp. 682-689). Hong Kong: Asia-Pacific Society for Computers in Education .

Roberts, E. S., Kassianidou, M., y Irani, L. (2002). Encouraging women in computer science. SIGCSE Bulletin, 34 (2), 84-88.

Shneiderman, B. (2007). Web science: a provocative invitation to computer science. Communications ACM, 50 (6), 25-27.

Sloan, R. H. y Troy, P. (2008). CS 0.5: A better approach to introductory computer science for majors. En Proceedings of the 39th SIGCSE Technical Symposium on Computer Science Education (pp. 271-275). Porland, Oregon, USA: ACM Press.

Vernor, V. (1993). The Coming Technological Singularity. Presentada en el Vision-21 Symposium, NASA Lewis Research Center and the Ohio Aerospace Institute, del 30 al 31 de marzo de 1993.

Vesgo, J. (2008). Enrollment and degree production at US CS departments drop further in 2006/2007. CRA Bulletin, 20 (2), p.p. 4.

Para citar el presente artículo puede utilizar la siguiente referencia:

Miraut Andrés, D. y Mendoza, A. (2011). Iniciativas educativas para una sociedad de la información sostenible. Revista Teoría de la Educación: Educación y Cultura en la Sociedad de la Información. 12(3), 241-264 [Fecha de consulta: dd/mm/aaaa]. http://campus.usal.es/ revistas_trabajo/index.php/revistatesi/article/view/8491/8584

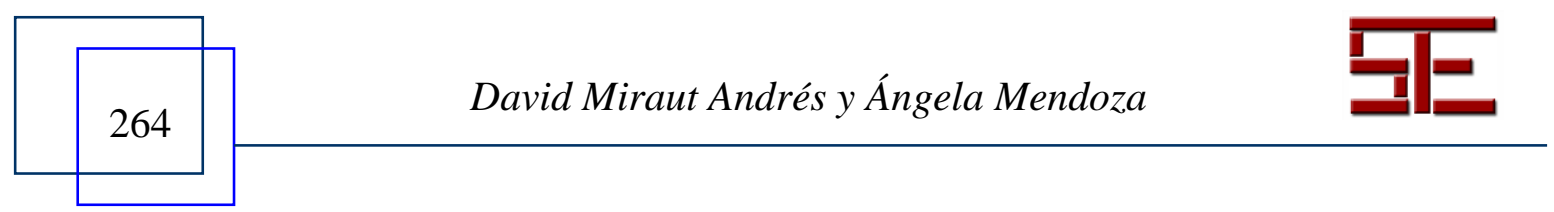

